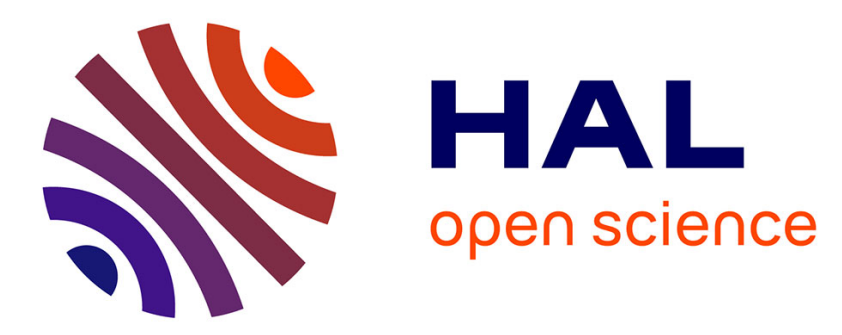

\title{
Measurement of Soret and Fickian diffusion coefficients by orthogonal phase-shifting interferometry and its application to protein aqueous solutions
}

Juan Felipe Torres, Atsuki Komiya, Daniel Henry, Shigenao Maruyama

\section{- To cite this version:}

Juan Felipe Torres, Atsuki Komiya, Daniel Henry, Shigenao Maruyama. Measurement of Soret and Fickian diffusion coefficients by orthogonal phase-shifting interferometry and its application to protein aqueous solutions. Journal of Chemical Physics, 2013, 139 (7), pp.074203. 10.1063/1.4817682 . hal00931501

\author{
HAL Id: hal-00931501 \\ https://hal.science/hal-00931501
}

Submitted on 11 Apr 2016

HAL is a multi-disciplinary open access archive for the deposit and dissemination of scientific research documents, whether they are published or not. The documents may come from teaching and research institutions in France or abroad, or from public or private research centers.
L'archive ouverte pluridisciplinaire HAL, est destinée au dépôt et à la diffusion de documents scientifiques de niveau recherche, publiés ou non, émanant des établissements d'enseignement et de recherche français ou étrangers, des laboratoires publics ou privés. 


\title{
Measurement of Soret and Fickian diffusion coefficients by orthogonal phase-shifting interferometry and its application to protein aqueous solutions
}

\author{
Juan F. Torres, 1,2,a) Atsuki Komiya,, ${ }^{3, b)}$ Daniel Henry, ${ }^{2, c)}$ and Shigenao Maruyama ${ }^{3}$ \\ ${ }^{1}$ Graduate School of Engineering, Tohoku University, 6-6-04, Aramaki Aza Aoba Aoba-ku, Sendai, Miyagi \\ 980-8579, Japan \\ ${ }^{2}$ Laboratoire de Mécanique des Fluides et d'Acoustique, CNRS/Université de Lyon, École Centrale de \\ Lyon/Université Lyon 1/INSA de Lyon, ECL, 36 avenue Guy de Collongue, 69134 Ecully Cedex, France \\ ${ }^{3}$ Institute of Fluid Science, Tohoku University, 2-1-1, Katahira, Aoba-ku, Sendai, Miyagi 980-8577, Japan
}

(Received 9 May 2013; accepted 23 July 2013; published online 20 August 2013)

\begin{abstract}
We have developed a method to measure thermodiffusion and Fickian diffusion in transparent binary solutions. The measuring instrument consists of two orthogonally aligned phase-shifting interferometers coupled with a single rotating polarizer. This high-resolution interferometer, initially developed to measure isothermal diffusion coefficients in liquid systems [J. F. Torres, A. Komiya, E. Shoji, J. Okajima, and S. Maruyama, Opt. Lasers Eng. 50, 1287 (2012)], was modified to measure transient concentration profiles in binary solutions subject to a linear temperature gradient. A convectionless thermodiffusion field was created in a binary solution sample that is placed inside a Soret cell. This cell consists of a parallelepiped cavity with a horizontal cross-section area of 10 $\times 20 \mathrm{~mm}^{2}$, a variable height of 1-2 $\mathrm{mm}$, and transparent lateral walls. The small height of the cell reduces the volume of the sample, shortens the measurement time, and increases the hydrodynamic stability of the system. An additional free diffusion experiment with the same optical apparatus provides the so-called contrast factors that relate the unwrapped phase and concentration gradients, i.e., the measurement technique is independent and robust. The Soret coefficient is determined from the concentration and temperature differences between the upper and lower boundaries measured by the interferometer and thermocouples, respectively. The Fickian diffusion coefficient is obtained by fitting a numerical solution to the experimental concentration profile. The method is validated through the measurement of thermodiffusion in the well-known liquid pairs of ethanol-water (ethanol $39.12 \mathrm{wt} . \%)$ and isobutylbenzene-dodecane (50.0 wt.\%). The obtained coefficients agree with the literature values within 5.0\%. Finally, the developed technique is applied to visualize biomolecular thermophoresis. Two protein aqueous solutions at $3 \mathrm{mg} / \mathrm{ml}$ were used as samples: aprotinin $(6.5 \mathrm{kDa})$-water and lysozyme $(14.3 \mathrm{kDa})$-water. It was found that the former protein molecules are thermophilic and the latter thermophobic. In contrast to previously reported methods, this technique is suitable for both short time and negative Soret coefficient measurements. () 2013 AIP Publishing LLC. [http://dx.doi.org/10.1063/1.4817682]
\end{abstract}

\section{INTRODUCTION}

Thermodiffusion, also called Soret effect, is the diffusion of molecules under a temperature gradient. ${ }^{1,2}$ This phenomenon induces a separation of species, as opposed to the mixing of species that occurs in Fickian diffusion. ${ }^{3-5}$ Thermodiffusion is of great importance in various fields. In the petroleum industry, for example, thermodiffusion determines the distribution of hydrocarbon mixtures in oil reservoirs. ${ }^{6}$ In biotechnology, thermodiffusion (also called thermophoresis ${ }^{7}$ ) has been proposed as an alternative method to electrophoresis for separation of biomacromolecules, such as proteins and DNA. ${ }^{8}$ Thermodiffusion also provides information about proteins concerning their molecular size, charge, and hydration

\footnotetext{
a)Electronic mail: torres.jf@ outlook.com

b) Electronic mail: komy@pixy.ifs.tohoku.ac.jp

c)Electronic mail: daniel.henry@ec-lyon.fr
}

shell, ${ }^{9}$ i.e., it has a strong potential for drug screening applications as well as basic research. ${ }^{10}$ Therefore, various experimental techniques have been developed with the purpose of quantifying the Soret effect. ${ }^{11}$

Most of the measuring techniques have been developed focusing on transparent binary solutions without the presence of biomolecules, such as proteins. Several European research groups established the Fontainebleau benchmark in a concerted effort to provide reference values for Soret, diffusion, and thermodiffusion coefficients. ${ }^{11-13}$ This benchmark accounts for three binary mixtures formed at 50 wt. $\%$ concentration and $25^{\circ} \mathrm{C}$ temperature by pairing three organic compounds: 1,2,3,4-tetrahydronaphthalene (THN), isobutylbenzene (IBB), and dodecane (C12). These compounds were chosen based on their relevance in oil reservoirs. On the other hand, Iacopini and Piazza ${ }^{14}$ have conducted some of the few systematic measurements of thermodiffusion in protein solutions. Piazza et al. ${ }^{15}$ discussed the physical 
mechanisms underlying thermophoresis in colloidal suspensions and macromolecular solutions, and presented a general model suggesting thermophoretic measurements as a sensitive probe of particle-solvent interfacial properties. They reported the Soret coefficient of hen egg-white lysozyme solutions at different temperature, $\mathrm{pH}$, and ionic strengths. Moreover, some recent studies have focused on thermodiffusion in ternary mixtures as a first step to clarify its mechanism in multicomponent systems. ${ }^{16}$ Hence, it is necessary to develop a robust experimental technique that is capable of providing insight into macromolecular thermodiffusion in binary systems, with the aim of a further application in more realistic multicomponent environments.

Even though theoretical correlations have been proposed to predict the thermodiffusion phenomenon, ${ }^{17}$ complex molecular interactions have made these approaches a rather ambiguous approximation to specific molecular characteristics. This is another reason to improve the current experimental techniques, so that accurate data is obtained for further theoretical development. All the techniques that have been developed to measure thermodiffusion can be classified according to the heating configuration and the method for measuring concentration. There are two groups regarding the heating configuration: convectionless methods and methods with convective coupling. ${ }^{11}$ Likewise, there are two groups regarding the concentration measurement method: chemical analysis and refractive index measurement.

The convectionless methods were the first methods to be developed (in fact, one of these methods was used by Soret to discover the thermodiffusion phenomena ${ }^{2}$ ). They consist on placing a binary solution inside a cavity and heating it such that the denser region of the solution resides on the bottom of the cell and, thus, creating a hydrodynamic stable system without convection. Convectionless methods usually make use of a standard Soret cell, which consists of a parallelepiped cavity with two horizontal rigid plates made of a good thermal conductive material, e.g., copper or stainless steel. The plates are maintained at different constant temperatures by a thermostatic circulating coolant ${ }^{18}$ or Peltier modules, ${ }^{16,19}$ the latter becoming the norm in recent years to achieve accurate and fast temperature control. The solution is usually heated from above to avoid natural convection. However, there are ranges of negative Soret coefficients when heating from below has a stabilizing effect ${ }^{20}$ in comparison to heating from above with the same temperature difference.

In contrast to convectionless methods, convective coupling methods are those in which the sample is subject to mass transport due to both natural convection and thermodiffusion. The Rayleigh-Bénard configuration has been used to determine the Soret coefficient. ${ }^{21}$ Thermogravitational column ${ }^{22,23}$ is another well-known method where a horizontal heat flux is applied to the sample. It is still a matter of debate to know which heating configuration, convectionless or with convective coupling, is the most adequate to measure Soret coefficients. Nevertheless, it is clear that in convectionless binary systems the measurement of the Soret coefficient is straightforward after the steady states for mass and heat have been reached. ${ }^{11}$
Concerning the measurement method of the concentration, the optical techniques are regarded as the most accurate, ${ }^{24}$ providing reliable measurements of the Soret coefficients. ${ }^{25}$ All optical techniques use the relation between the refractive index and the concentration - involving the so called contrast factors ${ }^{26}$ - in order to conduct temporal measurements of concentration either in a localized point, e.g., in the case of the optical beam deflection (OBD) technique, or in a two-dimensional domain, e.g., in the case of the interferometric techniques. Furthermore, in addition to the probe laser beam used for concentration measurements, Thermal Diffusion Forced Rayleigh Scattering (TDFRS) ${ }^{27,28}$ and thermal lensing ${ }^{29,30}$ make use of a high intensity laser in order to introduce a temperature gradient within the sample. TDFRS has been widely used to determine Soret coefficients in polymers and colloids. ${ }^{31-33}$ Nonetheless, in conventional TDFRS the use of an additive is required to absorb heat in order to increase the temperature of the sample, resulting in an undesired contamination that might affect the transport properties of the species of interest. Therefore, to avoid the drawbacks of using a third component, techniques with in situ optical detection coupled with a boundary temperature control system have been developed, e.g., $\mathrm{OBD}^{13}$ and interferometric techniques. ${ }^{20,34}$ Even though OBD is one of the most reliable methods currently available to determine Soret coefficients, it is a localized measurement that does not take into account any $2 \mathrm{D}$ or $3 \mathrm{D}$ characteristics of the thermodiffusion phenomena. In this study, therefore, we have focused on interferometric techniques in order to deepen our understanding of thermodiffusion.

Colombani et al. ${ }^{20}$ used holographic interferometry to study hydrodynamic instabilities and the Soret effect in aqueous electrolytes. Mialdun and Shevtsova ${ }^{34,35}$ developed a Mach-Zehnder interferometer coupled with an image processing technique based on a fast Fourier-transform ${ }^{36}$ in order to obtain a high-resolution phase diagram instead of the conventional interferogram. This method, termed optical digital interferometry (ODI), has drastically increased the accuracy of the interferometric measurement. ODI was validated with the Fontainebleau benchmark. ${ }^{19}$ The technique was subsequently used to measure thermodiffusion in water-isopropanol mixtures in the International Space Station, ${ }^{25}$ and investigate the influence of vibrations on the diffusion in liquids. ${ }^{37,38}$ In space, ODI from two perpendicular views was used to follow a component separation $\Delta C$ of less than $1 \%$ of its initial mean value. In these experiments, however, the measurement time is rather long, e.g., about $18 \mathrm{~h}$ for the space experiments. A shorter measurement time is desirable, mainly for three reasons. First, microgravity experiments are costly; second, conformational changes might occur in biomolecular solutions altering its Soret coefficient value; third, the experimental conditions are more difficult to control. Furthermore, in real applications, a smaller amount of sample is also desirable in order to decrease the measurement costs, which increase drastically for rare biomolecules.

On the other hand, our research group has developed several temporal phase-shifting techniques in order to determine isothermal diffusion coefficients in binary solutions. ${ }^{39-42} \mathrm{We}$ have focused on reducing the measurement time by using a 
counter flow type cell ${ }^{41}$ and increasing the accuracy of the measurement by using phase-shifting interferometry. ${ }^{40}$ Our most recent work ${ }^{42}$ makes use of a phase-shifting interferometer coupled to a rotating polarizer in order to conduct accurate measurements of mass diffusion fields. The transient concentration profiles were then compared by inverse analysis to a numerical calculation based on Fick's law in order to determine the concentration dependency of mass diffusion coefficients in isothermal conditions. This technique was used to determine the isothermal diffusion coefficient of seven different proteins in a dilute aqueous medium, ${ }^{43}$ and was also used in dilute ternary systems. ${ }^{44}$

The objectives of this study are twofold:

(1) Modify the interferometric technique in order to measure Soret coefficients in transparent binary solutions. A new Soret cell is designed to shorten the measurement time and reduce the amount of sample, while preserving its convectionless state. The technique is validated by comparison with benchmark values.

(2) Use the measurement technique to visualize thermodiffusion in protein aqueous solutions. The Soret and Fickian diffusion coefficients are reported.

This experimental technique would provide a new optical tool to deepen our understanding of biomolecular thermodiffusion.

\section{EVALUATION METHOD}

The Soret coefficient $S_{T}$ in binary systems is defined as

$$
S_{T}=\frac{D_{T}}{D},
$$

where $D_{T}$ and $D$ are the thermodiffusion coefficient and isothermal diffusion coefficient, respectively, which both enter the mass flux equation defined as

$$
J_{C}=-\rho D \nabla C-\rho C_{0}\left(1-C_{0}\right) D_{T} \nabla T,
$$

where $J_{c}$ is the mass flux, $\rho$ is the total density, $T$ is the temperature, $C$ is the concentration of the considered component expressed as the mass fraction (non-dimensional), and $C_{0}$ is the mean concentration of one species inside the differential control volume. The weight factor $C_{0}\left(1-C_{0}\right)$ is associated with $D_{T}$ to define the constant of proportionality between mass flux and temperature gradient. The weight factor indicates that thermodiffusion does not exist in pure fluids, i.e., $C_{0}\left(1-C_{0}\right)$ $=0$, but as a matter of fact the thermodiffusion coefficient $D_{T}$ usually has a finite value in the infinite dilute region. In contrast, $D$ is simply defined as the phenomenological constant of proportionality between mass flux and concentration gradient. Each coefficient $D_{T}$ and $D$ is defined in accordance with the laws of mass diffusion that were discovered in the experimental works reported by Soret ${ }^{2}$ and Graham, ${ }^{3,4}$ respectively.

If a binary solution is placed inside a Soret cell that has adiabatic sidewalls and a constant temperature difference $\Delta T$ between its upper and lower walls, a constant concentration difference $\Delta C$ between the upper and lower boundaries is reached after an infinite time when $S_{T} \neq 0$. In this steady state, the mass flux becomes zero $\left(J_{c}=0\right)$ and from Eqs. (1) and (2), the Soret coefficient in a binary system is obtained by

$$
S_{T}=-\frac{1}{C_{0}\left(1-C_{0}\right)} \frac{\Delta C}{\Delta T} .
$$

Here, $S_{T}$ is usually a concentration and temperature dependent thermophysical property with a positive or negative value. Therefore, Eq. (3) is only valid for small differences of concentration and temperature. Equation (3) indicates that $S_{T}$ is positive when the considered molecules migrate to the cold region, i.e., this species is thermophobic, and $S_{T}$ is negative when the considered molecules migrate to the hot region, i.e., this species is thermophilic. In a binary mixture, the two components have related concentrations and opposite Soret coefficients. It is then enough to express the results with regard to one of the components, considered as the reference component. Note that the denser component is often chosen as reference component. Figure 1 shows a typical stability diagram of a binary system ${ }^{45,46}$ enclosed in a Soret cell in terms of the critical Rayleigh number and the Soret coefficient corresponding to the denser component. The Rayleigh number is defined as

$$
R a=\frac{g \beta h^{3}\left(T_{\text {lower }}-T_{\text {upper }}\right)}{\nu \alpha},
$$

where $g$ is the acceleration of gravity, $\beta$ is thermal expansion coefficient, $h$ is the height of the cell, $v$ is the kinematic viscosity, $\alpha$ is the thermal diffusivity, $T_{\text {lower }}$ and $T_{\text {upper }}$ are the lower and upper boundary temperatures, respectively. With this choice, $R a$ is positive when the net heat flux is upward, i.e., the sample is heated from below. The critical Rayleigh number $R a_{c}$ indicates the threshold for convection to set in. As shown in Fig. 1, a stable convectionless state is always achieved by heating a thermophobic $\left(S_{T}>0\right)$ denser component from above $(R a<0)$. This situation corresponds to that

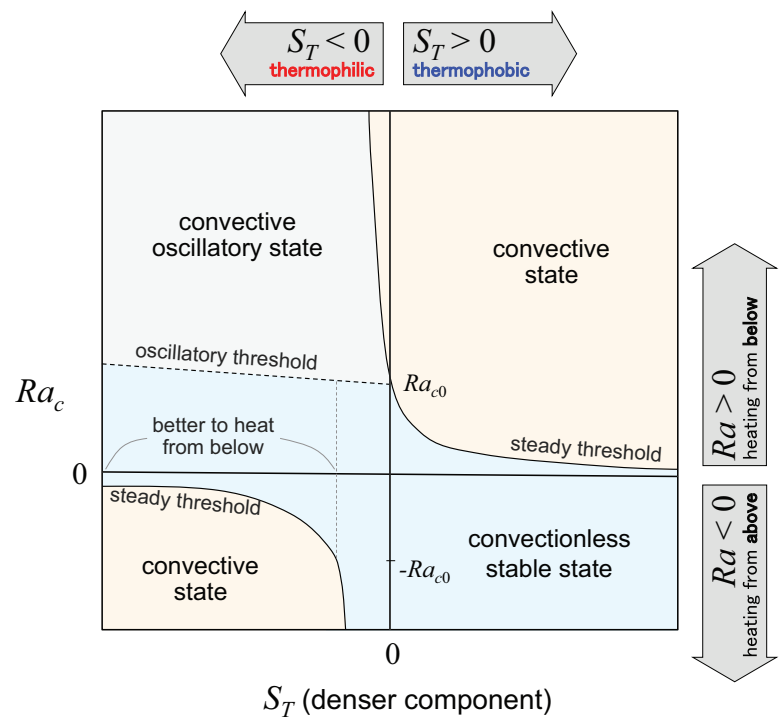

FIG. 1. Sketch of the stability diagram of a binary solution inside a Soret cell expressed in terms of the critical Rayleigh number $R a_{c}$ as a function of the Soret coefficient $S_{T}$ of the denser component. ${ }^{45,46}$ The configuration heated from above $(R a<0)$ corresponds to the one used in most conventional convectionless methods to measure the Soret coefficient. 
used in most convectionless methods recently developed. ${ }^{16,19}$ However, as indicated in Fig. 1, heating from below $(R a>0)$ creates a more stable state than heating from above for a binary solution with a thermophilic denser component when $\left|S_{T}\right|$ is sufficiently high. Melnikov et al. ${ }^{47}$ conducted 3D calculations on Soret driven instability of convective flows in cavities heated from above with negative $S_{T}$.

In this study, we aim to conduct an active control of the temperature between the upper and lower boundaries of a Soret cell, i.e., controlling $\Delta T$, and perform an interferometric measurement of the concentration difference in a solution between the upper and lower liquid/wall boundaries, i.e., measuring $\Delta C$. The binary solution used in each experiment is prepared at the desired concentration $C_{0}$. The Soret coefficient $S_{T}$ is then determined by substituting $\Delta T, \Delta C$, and $C_{0}$ in Eq. (3).

Furthermore, the mass diffusion coefficient is determined from the isothermal diffusion experimental phase. The transient isothermal concentration profile $C_{\exp }(z, t)$, which is measured with the interferometer, is recorded in order to fit a numerical calculation based on Fick's law $C_{c a l}(z, t)$ to this experimental result; here, $z$ is the coordinate in the direction of the heat flux inside the Soret cell (vertical direction). The fitting process, conducted by inverse analysis, yields the Fickian diffusion coefficient of the binary solution at an isothermal temperature $T_{m}$ and mean concentration $C_{0}$. The objective function $\delta$ is defined as the area between $C_{\exp }(z, t)$ and $C_{c a l}(z, t)$ and is written as

$$
\delta\left(D_{c a l}\right)=\sum_{i}^{k}\left|C_{\exp }\left(z_{i}, t_{r e f}\right)-C_{c a l}\left(z_{i}, t_{r e f}, D_{c a l}\right)\right| \Delta z,
$$

where $D_{c a l}$ is the isothermal diffusion coefficient used in the numerical calculation, $t_{\text {ref }}$ is the reference time, $\Delta z$ is the vertical distance between two neighboring pixels, and $k$ is the total number of pixels in the mass flux direction. The numerical calculation uses the experimental concentration profile $C_{\exp }\left(z, t_{i n i}\right)$, where $t_{i n i}<t_{\text {ref }}$, as initial condition. Since the objective function is unimodal, a simple one-dimensional search can be executed to minimize $\delta$ and, thus, determine the isothermal diffusion coefficient $D$. In this study, we used the golden section search to minimize Eq. (5). The thermodiffusion coefficient $D_{T}$ can then be determined by substituting $S_{T}$ and $D$ in Eq. (1).

\section{EXPERIMENT}

\section{A. Experimental apparatus}

A new experimental setup was designed to study thermodiffusion in transparent liquid systems. The instrument consists of two main parts: the Soret cell where the thermodiffusion field is created, and the optical system that is used to measure transient concentration profiles.

\section{Soret cell}

The Soret cell designed in this study is shown in Fig. 2. The binary solution is placed inside a rectangular parallelepiped cavity with a horizontal cross-section area of $10 \times 20 \mathrm{~mm}^{2}$ and a variable height of $1-2 \mathrm{~mm}$, as shown in the lateral and horizontal cross-section views in Figs. 2(b) and 2(c). The upper and lower boundaries are made of copper, which has a high thermal conductivity so that a rapid temperature control is achieved. The lateral walls are made of quartz to allow the passage through the sample of two orthogonal laser beams, i.e., test beams $L 1$ and $L 2$. The optical setup is described in Sec. III A 2.

A standard quartz cell with high optical quality (Fine Inc., $\mathrm{W} \times \mathrm{L} \times \mathrm{H}=10 \times 20 \times 45 \mathrm{~mm}^{3}$ ) was cut into a bottomless four-sided quartz glass that was subsequently clamped between two thermostabilized copper blocks, as shown in Fig. 2(a). The quartz glass is attached to the copper blocks by a thermoplastic adhesive that can be removed after finishing the experiment. This avoids leaks and allows the detachment of the quartz part for cleaning and further use. Moreover, two holes of $1 \mathrm{~mm}$ in diameter were drilled near the edges of the cell with a double purpose: to inject the binary

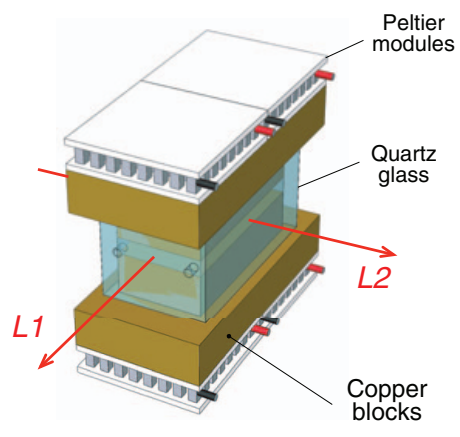

(a)

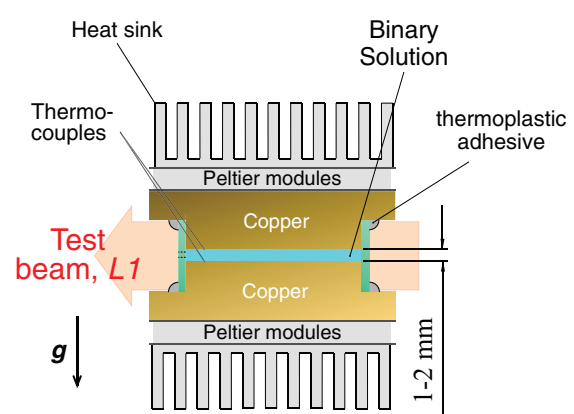

(b)

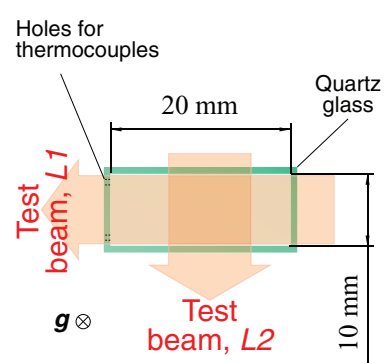

(c)

FIG. 2. The Soret cell where the binary solution is injected. (a) Three-dimensional model of the cell, (b) its lateral cross-section, and (c) a horizontal crosssection view of the cavity where the sample is placed. The lateral walls of the cell are transparent to allow the passage of two orthogonal test beams. The temperature control of the upper and lower copper boundaries is conducted by Peltier modules integrated to a PID system. Two holes were drilled in a quartz wall to fill the cavity with the solution while avoiding bubbles, and to introduce the thermocouples in order to measure the temperature just at the copper/liquid boundary. 
solution, and to introduce the thermocouples that are used to measure the temperatures of the upper and lower boundaries inside the cell. In order to avoid bubbles, the binary solution is injected through one hole using a micro-syringe (Hamilton Company, $500 \mu \mathrm{m}$ ) while removing the air from the other hole; this procedure is conducted with the cell turned $90^{\circ}$ with its holes facing upwards to facilitate the exit of air. It is imperative that the binary solution entirely fills the cell without leaving any bubbles; a small bubble could grow within the cell due to evaporation of the solvent into the surrounding air through the glass holes, blocking the optical beam used in the measurement. In any case, the cell is sealed with a thermoplastic adhesive to avoid contact with the surrounding air in experiments using highly volatile liquids, such as ethanol, because an open cell might also result in undesired concentration gradients near the free boundaries due to evaporation. On the other hand, it is not necessary to seal the cell when the binary solution has low volatility, as in the case of diluted protein aqueous solutions. Not sealing the cell guarantees that the pressure inside the cavity is kept at atmospheric pressure regardless of the thermal expansion of the liquid and its cell boundaries. Nevertheless, the effects of the thermal expansion are negligible in these experiments because the temperature difference is small. A large temperature difference would not only affect the hydrostatic pressure, but might also result in a rupture of the quartz walls due to the thermally induced stress.

Mialdun and Shevtsova ${ }^{19}$ took extra precautions to avoid bubbles by degassing the sample. They used a degasser designed for solvents in high performance liquid chromatography (HPLC) applications. In this study, the use of a degasser is less relevant because the measurement time is about $1 / 10$ of that reported in the experiments of Mialdun and Shevtsova ${ }^{19}$

a. Rapid temperature control. The temperature control of the upper and lower boundaries is conducted by a single computer-driven PID system, enabling temperature stability of $\pm 0.01 \mathrm{~K}$. Takeda et al $^{48}$ used a similar PID setup in a cryoprobe that is used in safe and reliable cryosurgery. The temperature at the copper/liquid boundaries inside the Soret cell is measured by T-type thermocouples. These were calibrated inside iced water at $0{ }^{\circ} \mathrm{C}$. The PID system controls the mean temperature $T_{m}$ within the cell and the temperature difference $\Delta T$ between the upper and lower copper/liquid boundaries. Figure 3 shows the temperature control at the boundaries for a typical thermodiffusion experiment. Here, $T_{m}=25^{\circ} \mathrm{C}, \Delta T$ $=5^{\circ} \mathrm{C}$, and the distance between the copper walls is set to $h$ $=2 \mathrm{~mm}$. The resulting overshoot $\delta T^{\prime}$ and settling time $t_{s}$ for these conditions are $0.71{ }^{\circ} \mathrm{C}$ and $44 \mathrm{~s}$, respectively.

The type of heat sink used determines the controllable temperature range of the Peltier modules. Coolant exchangers are preferable as a heat sink for large temperature differences or a long experimental time. ${ }^{19}$ The use of liquid nitrogen as a heat sink is the best choice for temperatures lower than $0{ }^{\circ} \mathrm{C} .{ }^{48}$ In this set of experiments, standard fins were employed since the temperature ranges inside the cell are within $\pm 5^{\circ} \mathrm{C}$ of the room temperature $T_{\text {room }}$ and the experimental time is within a couple of hours. The use of fins as heat sink provides a simple setup without moving components that might

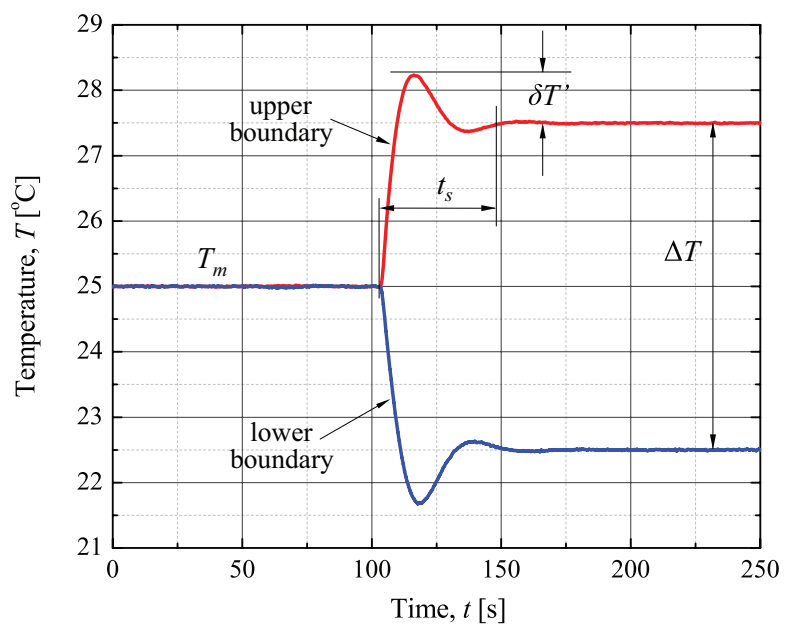

FIG. 3. PID temperature control at the copper/liquid boundaries. The temperature is measured just at the liquid interface by extra thin T-type thermocouples. The temperature control for the upper and lower boundaries is simultaneously performed to set a constant temperature difference $\Delta T$ and a mean temperature $T_{m}$. The overshoot $\delta T^{\prime}$ and settling time $t_{s}$ depend on PID gains chosen in the control routine.

affect the optical measurement, which is very sensitive to vibrations.

b. Design parameters. Similar Soret cells to that shown in Fig. 2 have been used in interferometric measurements. Colombani et al. ${ }^{20}$ used a cell with cavity dimensions of $\mathrm{W} \times \mathrm{L} \times \mathrm{H}=10 \times 10 \times 30 \mathrm{~mm}^{3} ;$ Mialdun and Shevtsova ${ }^{19}$ used a cell with dimensions $\mathrm{W} \times \mathrm{L} \times \mathrm{H}=22 \times 22 \times 6.3$ $\mathrm{mm}^{3}$. However, the measurement time reported by these research groups was around $200 \mathrm{~h}$ and $15 \mathrm{~h}$, respectively. The characteristic measurement time $\tau$ of the thermodiffusion experiment is bound to the strength of the Fickian diffusion (isothermal diffusion coefficient $D$ ) and to the diffusion length $h$ as follows

$$
\tau=\frac{h^{2}}{\pi^{2} D}
$$

Therefore, decreasing the height of the cell $h$ would drastically reduce the measurement time for a given Fickian diffusion coefficient. Equation (6) also indicates that for macromolecules, which have a smaller $D$ as predicted by the Stokes-Einstein equation, the measurement time increases. This makes the measurement of thermodiffusion in biological systems a more difficult task since most biomolecules, such as proteins, are macromolecules with a diffusion coefficient in the order of $10^{-10}-10^{-11} \mathrm{~m}^{2} / \mathrm{s}$. Furthermore, as indicated by Eq. (4), decreasing $h$ would drastically increase the stability of the system while preserving the heating-from-above configuration $(R a<0)$. This is of particular importance in measurements where the sign of the Soret coefficient is unknown. Therefore, for our measurements in protein solutions, it is important to choose a cell with a small height. Such cell bears more resemblance to those cells used in OBD studies, as in the experiment described by Königer et al. ${ }^{16}$ 
In this study, we focus on designing a Soret cell with a short height within the optical constrains. The small height $h$ reduces the sample volume (Volume $\propto h$ ), shortens the measurement time ( $\tau \propto h^{2}$, see Eq. (6)), and increases the hydrodynamic stability of the system $\left(R a \propto h^{3}\right.$, see Eq. (4)). The optical problems related to a short $h$ will be further discussed.

\section{Orthogonal phase-shifting interferometry}

The optical system consists of two orthogonally aligned Mach-Zehnder phase-shifting interferometers; the technique is, therefore, termed orthogonal phase-shifting interferometry (OPSI). Two independent beams with the same wavelength permit different sensitivities, which are determined by the optical path (OP) inside the cell (see Fig. 2). The sensitivity of the interferometers may also be varied by changing the laser wavelength. ${ }^{16}$ We have previously used a single phase-shifting interferometer to visualize diffusion fields in isothermal conditions. ${ }^{42}$ The phase-shifting technique was previously validated by measuring the concentration dependence of the diffusion coefficient in two well-known binary systems, $\mathrm{NaCl}$-water and sucrose-water at $25^{\circ} \mathrm{C}$. A brief description of the modified interferometric layout, the real-time image processing, and the post experimental image processing is presented in the following.

a. Interferometric layout. The interferometric layout is shown in Fig. 4. This optical device was built on a high-performance vibration isolation system (Herz Co., TDI-2010LAY) and placed inside an enclosed room environmentally controlled at room temperature $T_{\text {room }}\left( \pm 0.5^{\circ} \mathrm{C}\right.$ uncertainty) and low humidity. For a quasi-one-dimensional phenomenon, such as the diffusion and thermodiffusion fields created in this study, only one interferometric view would suffice. However, the introduction of an additional interferometer allows us to have two different optical paths for the test beams within the Soret cell, and thus, different sensitivities can be selected in order to visualize two different phenomena such as heat conduction and mass diffusion. In this study, both interferometers are used to visualize mass diffusion. The interferometer with the laser beam $L 1$ is used during the thermodiffusion phase $(\Delta T \neq 0)$, while the interferometer with the laser beam $L 2$ is used during the isothermal diffusion phase $(\Delta T=0)$.

Two Helium-Neon laser beams (L1- Newport Corp., $\lambda=633 \mathrm{~nm}, 5.0 \mathrm{~mW}$, and $L 2-$ Neoark Corp. $\lambda=632.8 \mathrm{~nm}$, $7.0 \mathrm{~mW}$ ) are linearly polarized by polarizers inclined by $\pi / 2$

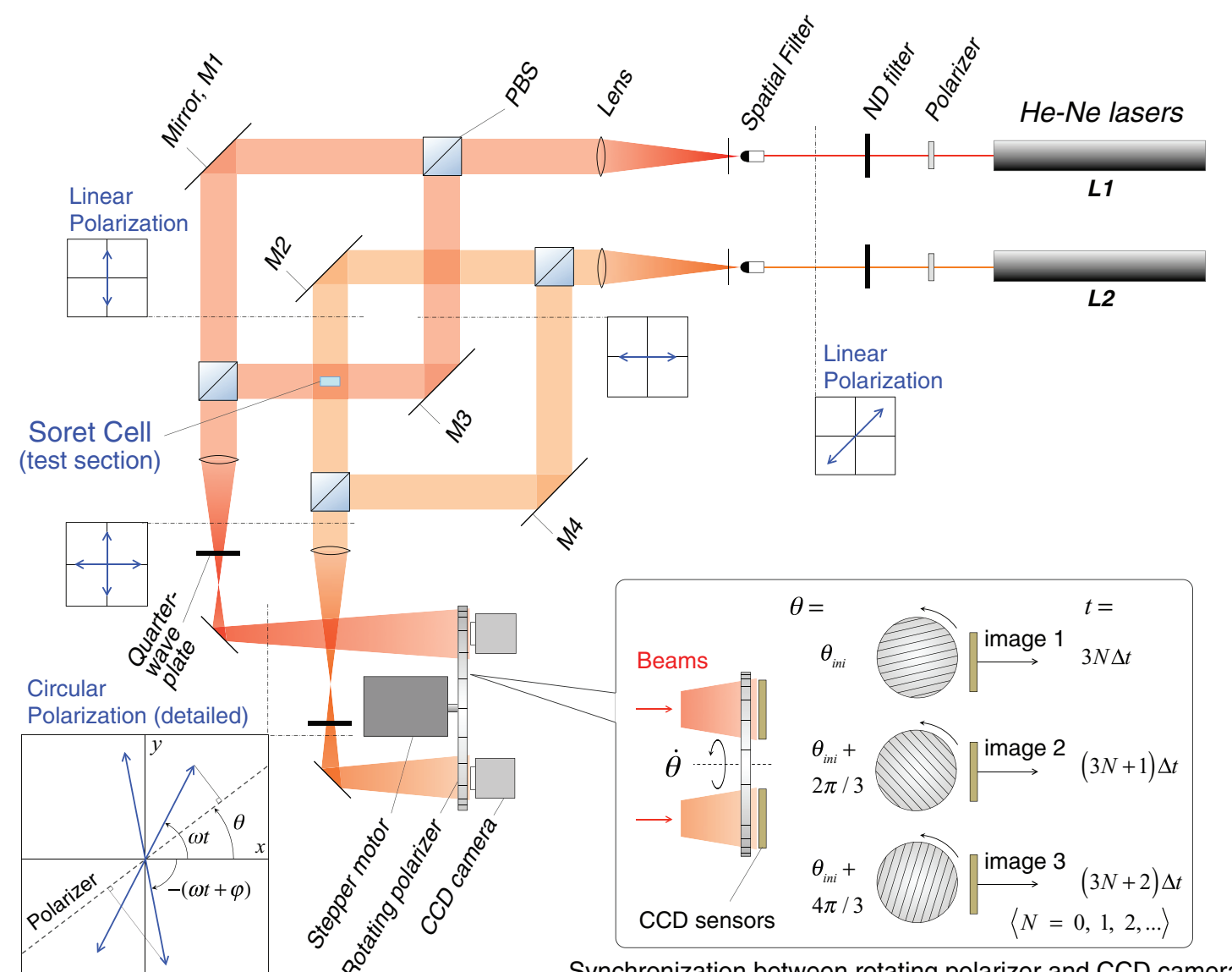

FIG. 4. Two orthogonally aligned phase-shifting interferometers. The polarization state of light is shown for each segment. The Soret cell is placed inside the interferometer to visualize thermodiffusion and isothermal diffusion from two orthogonal directions. The lower right inset shows the rotating polarizer, which is synchronized with the CCD camera in order to take interferograms at angle and time intervals of $\Delta \theta=2 \pi / 3$ and $\Delta t=1 / 30 \mathrm{~s}$, respectively. 
with respect to the horizontal. The intensity of each laser beam is adjusted using ND filters. Here, an independent intensity adjustment for each beam is necessary in the case the interferometers have different magnifying rates, laser power output, or CCD cameras. The beams are then expanded with spatial filters (Sigma-Koki Inc.) and collimated with convex lenses. Polarizing beam splitters (PBS) (Newport Corp. 10BC16PC.4) are then used to split the beams into a test beam and a reference beam with orthogonal polarization state. The test beams from lasers $L 1$ and $L 2$ have horizontal and vertical linear polarization, respectively; whereas the reference beams from lasers $L 1$ and $L 2$ have vertical and horizontal linear polarization, respectively. The polarization state of light for each segment of the interferometer is indicated by the square insets in Fig. 4. Each set of test beam and reference beam are then combined by another PBS and focused on a CCD camera (Hamamatsu Inc. C3077-70) by convex lenses. After the second PBS, the relative polarization angle between the beams is $\pi / 2$. In order to make these beams interfere, a quarter-wave plate is placed after each focusing lens. Here, the extraordinary axis of the quarter wave-plate is inclined by $\pi / 4$ with respect to the horizontal, such that this inclination is between the linear polarization states of the test beam and reference beam. Therefore, both beams become circularly polarized with opposite handedness, i.e., for $L 1$ ( $L 2)$, the reference (test) beam is clockwise circularly polarized, while the test (reference) beam is counter-clockwise circularly polarized, as shown in the detailed circular polarization state in Fig. 4. At this point, only a partial linear interference occurs since the beams still have orthogonal components. In order to filter the light in a single linear direction to achieve complete interference, a polarizer is placed with an angle $\theta$ just before the CCD cameras. The cameras acquire an image similar to that of a typical interferogram. A single polarizer in disk-form with diameter of $130 \mathrm{~mm}$ was used to polarize both interferometric beams.

In this setup, the phase-shifting technique requires that the incident ray of light charges the same capacitor (sensor) of the CCD array at any rotating angle $\theta$. Therefore, it is crucial that the rotating polarizer is as perpendicular as possible to the incident beam. Moreover, placing the CCD sensor as close as possible to the rotating polarizer is also a crucial factor because it reduces the beam displacement in an exiting tilted beam. In addition to this optical problem, a poor alignment of the polarizer generates undesired vibrations that might affect the highly sensitive optical system. Hence, in order to obtain a well-aligned setup that is free of vibrations, it is imperative that the polarizer is placed with its center of mass aligned to the axis of the stepper motor while preserving its perpendicularity to the optical axis and closeness to the CCD sensor.

b. Real-time image processing. A high-resolution phaseshifted data is acquired in real-time by conducting an image processing technique and synchronizing the rotation of the polarizer with the frame rate of the CCD camera, as shown in the lower right inset of Fig. 4. The frame rate of the CCD camera is $29.97 \mathrm{fps}$ with a $640 \times 480$ pixel resolution; the polarizer is then rotated at an angular speed of $20 \pi \mathrm{rad} / \mathrm{s}$ by using a five-phase stepper motor (Oriental Motor Co., RK569AMA).
Therefore, the interferograms are taken at relative angles of $\Delta \theta=2 \pi / 3$ and time intervals of $\Delta t=1 / 29.97 \mathrm{~s}$. Three consecutive interferograms, as shown in Fig. 5(a), are used to acquire the phase-shifted data.

In this study, a temporal phase-shifting technique is employed. ${ }^{49}$ We have chosen a three-bucked technique ${ }^{50}$ with an angle between intensity readings of $2 \pi / 3$. The phaseshifting equation is

$$
\varphi=\arctan \left(\sqrt{3} \frac{I_{2}-I_{3}}{I_{2}-2 I_{1}+I_{3}}\right),
$$

where $\varphi$ is the phase shift between the reference beam and the test beam, $I_{1}, I_{2}$, and $I_{3}$ are the intensities of light detected by the CCD sensor at rotating polarizer angles of $\theta_{i n i},\left(\theta_{i n i}\right.$ $+2 \pi / 3)$, and $\left(\theta_{i n i}+4 \pi / 3\right)$, respectively. Because of the nature of the arctangent function, Eq. (7) is a modulo $\pi$ calculation. To determine the phase modulo $2 \pi$, the signs of $\sin \varphi$ and $\cos \varphi$ must be examined to adjust the phase (see page 366 in Ref. 50). Figure 5(a) shows the raw interferogram intensities $I_{1}, I_{2}$, and $I_{3}$ and the derived phase-shifted data, which is also called wrapped phase map $\varphi(x, y)$. Equidistant and parallel phase-shifted data suggests that the wavefront of the test beam is planar. Furthermore, the tilt of the mirrors $M 1$ and $M 4$ (see Fig. 4) can be adjusted to achieve the infinite fringe condition, which corresponds to parallel wavefronts of the test beam and reference beam for each laser beam $L 1$ and $L 2$.

The detailed derivation process of Eq. (7) is described in the Appendix. Its comprehension is essential to understand the concept of the temporal phase-shifting interferometry proposed in this study and its measurement limitations. Here, the phase shift $\varphi$ is assumed to be constant within three consecutive interferograms. Therefore, this technique is only applicable to measure steady state phenomena or slow phenomena where $\Delta t / \tau \ll 1$, i.e., the time resolution of the phase-shifted data $(3 \times \Delta t)$ is much shorter than the characteristic time of the measured physical quantity, e.g., $\tau$ in Eq. (6). The phaseshifting equation is then applied to the whole pixel domain $(x$, $y$ ), and its results are displayed in quasi-real-time in an external monitor with a time lag of $0.1 \mathrm{~s}$. The phase-shifted data are stored with the purpose of further image processing after the experiment has finished.

c. Postexperimental image processing. One of the most important advantages of digital interferometry is the suppression of the optical defects of the apparatus, e.g., possible defects found in the mirrors, prisms, or Soret cell walls. This same advantage is found in the more conventional holographic interferometry, ${ }^{51}$ where the defects are suppressed by initially recording the interference pattern of the test beam and reference beam in a holographic plate, and then illuminating the same plate only with the test beam. In contrast, in digital interferometry an initial phase-shifted data is recorded with the Soret cell filled with a liquid of constant refractive index (i.e., homogeneous liquid without temperature or concentration gradients), which will be subsequently subtracted from the phase difference acquired during the thermodiffusion experiment.

The phase-shifted data shown in Fig. 5(a) are obtained without placing the Soret cell in the test section. The parallel 


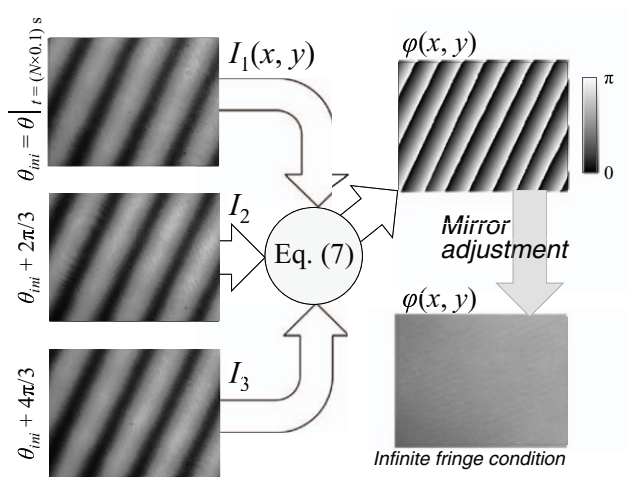

(a)

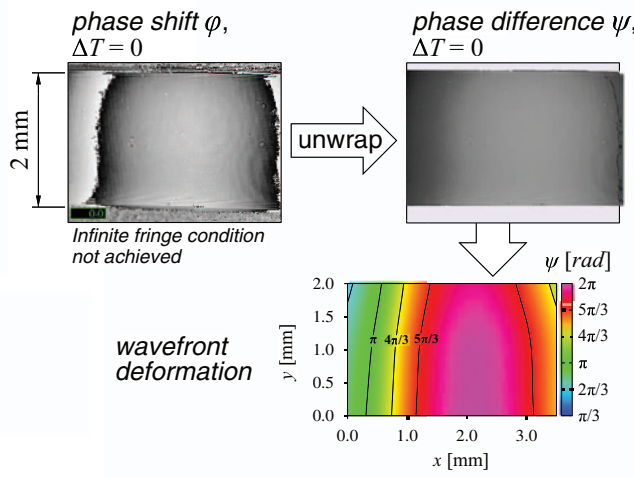

(b)

FIG. 5. Image processing of the interferograms in (a) quasi-real-time, and (b) post-experimental treatment. In the quasi-real-time image processing, three consecutive interferograms $I_{1}, I_{2}, I_{3}$, are treated with the phase-shifting equation, i.e., Eq. (7), in order to obtain the phase-shifted data $\varphi(x, y)$, which is also called wrapped phase map. An infinite fringe condition is achieved by adjusting the tilt of mirrors $M I$ and $M 4$ (see Fig. 4). However, the infinite fringe condition may not be achievable when placing the Soret cell in the test section due to possible optical defects of the quartz walls. These optical defects are translated into the unwrapped phase map that is a measurement of the deformed wavefront, as shown in (b). The unwrapped phase difference may be later used as background to obtain the real thermodiffusion field.

and equidistant fringes of the phase-shifted data indicate that there are virtually no deformations in our interferometric setup. However, the phase-shifted data shown in Fig. 5(b) indicates that the wavefront is curved when placing the Soret cell in the test section of the interferometer. Here, the cell is filled with a binary solution maintained at constant temperature and concentration (i.e., constant refractive index). The curved wavefront is, therefore, produced solely by the deformation of the quartz walls.

Figure 5(b) shows the unwrapped phase difference $\psi(x, y)$ that is obtained from the wrapped phase-shifted data $\varphi(x, y)$. This phase difference is an accurate measurement of the deformed wavefront. Furthermore, the initial unwrapped data are subtracted from the unwrapped data acquired during the thermodiffusion experiment in order to suppress the phase difference caused by the optical defects in the apparatus (including those of the quartz walls). This process yields the phase difference due to the refractive index variations caused solely by temperature and concentration gradients within the cell.

\section{B. Thermodiffusion experiment}

The measurement method of thermodiffusion coefficients is first validated by using two binary solutions wellreported in the literature: ethanol-water (ethanol 39.12 wt.\%) and isobutylbenzene-dodecane (50.0 wt.\%), the second solution being one of the three binary solutions used in the Fontainebleau benchmark. ${ }^{11}$ The technique is then applied to two diluted protein aqueous solutions at $3 \mathrm{mg} / \mathrm{ml}$ and $25^{\circ} \mathrm{C}$ : aprotinin $(6.5 \mathrm{kDa})$-water and lysozyme $(14.3 \mathrm{kDa})$-water. The lysozyme solution has been studied by Piazza et $a l .{ }^{15}$ at concentration $10 \mathrm{mg} / \mathrm{ml}$ for different temperatures, $\mathrm{pH}$, and ionic strengths. The Soret coefficient of the diluted lysozyme species is expected to be positive and close to zero at $\mathrm{pH}=7$ and $T=25^{\circ} \mathrm{C}$.

The binary solutions were prepared at a room temperature $T_{\text {room }}=25^{\circ} \mathrm{C}$. The concentration in the first two binary solutions is expressed in units of weigh percent [wt.\%] because each component is liquid in its pure form at $T_{\text {room }}$. On the other hand, the units of concentration $[\mathrm{mg} / \mathrm{ml}]$ are preferred in the case of the proteins solutions due to the solid state of the pure proteins at $T_{\text {room }}$. Protein molecules are known to fold when dissolved in water. ${ }^{52}$ The resulting molecular structure is defined by the ionic strength of the solution and, therefore, the diffusion coefficient is dependent on the ionic strength. Even though the thermophoretic mobility is a complex function of the surface particle potential and solution ionic strength, ${ }^{7}$ we have chosen to use two protein aqueous solutions in their highly aqueous diluted form as a first step to observe thermodiffusion of biomacromolecules.

Table I shows the name of the components used and their corresponding molecular mass, density (for those components in pure liquid form), and supplier with its lot number. All solutions were prepared in a sealed environment to avoid variation of the concentration due to vaporization. The proteins were diluted in distilled water and used in the delivered crystalized form without any further purification.

\section{Preliminary measurements}

Although a phase map resulting from refractive index variations due to concentration gradients is obtained with the optical system described above, we still need the corresponding $\Delta \psi / \Delta C$ ratio to obtain the concentration profile within the cell. Hence, preliminary measurements of the $\partial \psi / \partial C$ ratio are required. In the OBD studies, the ratio $\partial n / \partial C$ is used to couple the optical measurements with the concentration; here, $n$ is the refractive index. This ratio is usually termed contrast factor. Likewise, the ratio ${ }^{2} / \partial T$ also becomes relevant in non-isothermal experiments, such as in thermodiffusion experiments, because the refractive index is a temperature-dependent property. Nonetheless, the effects of $\partial n / \partial T$ on the wavefront can be optically suppressed in a thermodiffusion field where the temperature profile is linear within a small temperature difference. Therefore, in our set 
TABLE I. Components used in the four liquid pairs and the corresponding molecular mass and density (at $25^{\circ} \mathrm{C}$ ) of each pure liquid. Distilled water, dodecane, and isobutylbenzene are abbreviated as $\mathrm{H}_{2} \mathrm{O}, \mathrm{C} 12$, and IBB, respectively. The lot number and the suppliers, Wako Pure Chemical Industries (Wako), Ltd. and Sigma-Aldrich Co. (Sigma), are indicated. The proteins were supplied in crystalized form and, therefore, their density is omitted.

\begin{tabular}{lccc}
\hline \hline Component & Molecular mass (kDa) & Density (mg/ml) & Lot no. (supplier) \\
\hline Ethanol & 0.046 & 787 & DCN6248 (Wako) \\
Isobutylbenzene (IBB) & 0.134 & 853 & $\mathrm{H}_{2} \mathrm{O}$ \\
Dodecane (C12) & 0.170 & 780 & $\mathrm{C} 12$ \\
Aprotinin from bovine lung & 6.5 & $\ldots$ & DCM1071 (Wako) \\
Lysozyme from chicken egg-white & 14.3 & $\ldots$ & $058 \mathrm{~K} 7024(\mathrm{Sigma})$ \\
\hline \hline
\end{tabular}

of experiments only the $\partial \psi / \partial C$ ratio is required to measure the two-dimensional concentration field. The phase difference variation $\Delta \psi$ is linearly related to the refractive index variation $\Delta n$ as follows:

$$
\Delta n(x, z)=\frac{\lambda}{2 \pi L} \Delta \psi(x, z)
$$

where $L$ is the optical path in the liquid and $\lambda$ is the wavelength of the laser beam (fixed to $\lambda=632.8 \mathrm{~nm}$ in the current optical setup). The variation of the refractive index $\Delta n$ in Eq. (8) includes the contributions from both temperature and concentration ${ }^{19}$ when neither contribution is optically suppressed. The contribution of pressure is neglected since the pressure inside the cell is constant.

In spite of the importance of the contrast factors $\partial n / \partial C$, most measurements of thermodiffusion rely on contrast factors that are reported in the literature, while other studies develop independent measurement methods. In this study, we aim to conduct the Soret coefficient measurement with the same optical instrument through two experiments, providing a robust and independent measurement apparatus. Here, instead of the conventional ratio $\partial n / \partial c$, we measure the contrast factors $\partial \psi / \partial C$ with the phase-shifting interferometer.

The measurements of the contrast factors is done through isothermal diffusion experiments initially developed to determine isothermal mass diffusion coefficients. ${ }^{42}$ In contrast to our previous work, the measurement of Fickian diffusion coefficients is relegated to a secondary objective.

a. Contrast factors. A detailed explanation of the isothermal diffusion experiment is found in the literature. ${ }^{42}$ The same kind of experiment is performed to measure the contrast factors. In this section, important information concerning the data analysis is provided in addition to the one previously reported. ${ }^{42}$

In this set of experiments, a convectionless diffusion field is created by placing a lighter solution over a denser solution inside a diffusion cell and, as a consequence, a mass diffusion field triggered by concentration gradients is created. If the diffusion cell is transparent in its four lateral sides, the visualization of the diffusion field with OPSI is then possible.

Figure 6(a) shows the unwrapped phase shift between the lower and upper boundaries in an isothermal diffusion experiment using the binary system NaCl-water $(\Delta C=4 \mathrm{mg} / \mathrm{ml}$, $C_{\text {mean }}=2 \mathrm{mg} / \mathrm{ml}$ ). The insets show the phase-shifted data acquired at each corresponding time. The lower and upper

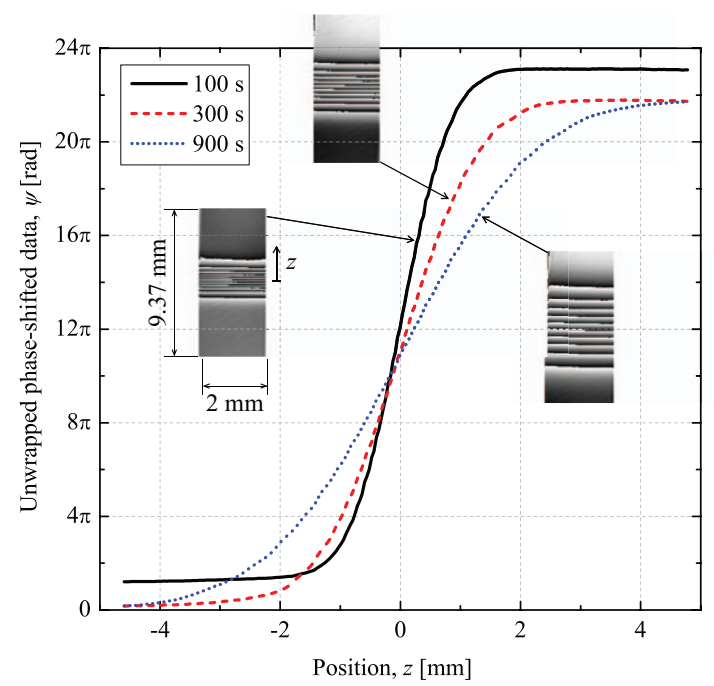

(a)

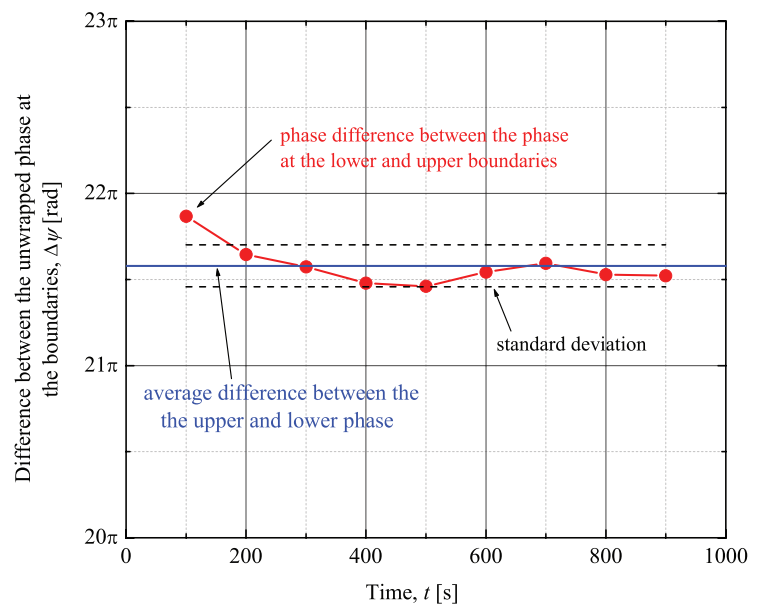

(b)

FIG. 6. Determination of $\Delta \psi / \Delta C$ for a dilute $\mathrm{NaCl}$ solution. (a) The unwrapped phase diagram obtained from the phase-shifted data shown in the insets. The phase diagram profile $\psi$ is the unwrapped data averaged in the horizontal direction perpendicular to $z$. (b) The phase difference $\Delta \psi$ between the upper and lower boundaries. The phase difference $\Delta \psi$ averaged in time is then divided by the concentration difference to obtain the ratio $\Delta \psi / \Delta C$. This value is used in the thermodiffusion experiments to determine the concentration profile within the Soret cell. 
visible boundaries at $z=-4.59 \mathrm{~mm}$ and $z=4.78 \mathrm{~mm}$, respectively, are immersed, i.e., Fickian diffusion through these boundaries can occur. The lateral vertical boundaries are impermeable. The data was averaged in the horizontal direction (isoconcentration direction perpendicular to $z$ ) after unwrapping. The intensity gradients between fringes in the phaseshifted data indicate that the phase difference is increasing with the vertical position $z$. Furthermore, the unwrapped phase shift profiles do not perfectly intersect at the center because the intensity of the phase-shifted data in the homogenous lower part of the solution varies at different times. This is due to temporal accumulative errors of the phase-shifting image processing. The proper boundary conditions are thus required in order to obtain the concentration profiles from each phase-shifted data. The phase difference between the lower and upper immersed boundaries of the unwrapped phase shift is shown in Fig. 6(b) as a function of time. The phase difference remains almost constant with a positive value $\Delta \psi_{\text {ave }}$ $=(21.58 \pm 0.12) \pi$ since there is no mass transport through the upper and lower observation limits, which correspond to immersed boundaries. If the fringes reach the upper or lower extremes, then there is diffusion through the corresponding immersed boundaries and, as a consequence, the phase difference $\Delta \psi$ is reduced. A homogenous solution is obtained when the experimental time tends to infinite, i.e., $\Delta \psi$ becomes zero. The transient concentration profile within the cell is obtained by introducing the corresponding concentration difference to each profile shown in Fig. 6(a).

In the trial experiment shown in Fig. 6(a), the average phase difference per units of concentration deduced from Fig. 6(b) is $\Delta \psi_{\text {ave }} / \Delta C=-(5.394 \pm 0.03) \pi\left[\mathrm{rad} \mathrm{ml} \mathrm{mg}{ }^{-1}\right]$. Moreover, the phase shift between the reference and test beams is directly proportional to the optical path $(\mathrm{OP})$ within the cell for a constant refractive index. As a consequence, the phase difference between the upper and lower boundaries is also directly proportional to the OP. In this study, we characterize the influence of the concentration difference $(\Delta C)$ and the $\mathrm{OP}(L)$ on the refractive index by using the following contrast factor:

$$
\eta=\frac{\Delta \psi}{\Delta C L}
$$

In the case of the trial experiment shown in Fig. 6(a), $\eta$ $=-(0.539 \pm 0.003) \pi\left[\mathrm{rad} \mathrm{ml} \mathrm{mg}{ }^{-1} \mathrm{~mm}^{-1}\right]$. The units for the concentration could be either $[\mathrm{mg} / \mathrm{ml}]$ or $[\mathrm{wt} . \%]$ depending on the type of the components involved. Substituting $\Delta \psi$ in Eq. (8) yields

$$
\left(\frac{\partial n}{\partial C}\right)_{p, T}=\frac{\lambda \eta}{2 \pi} .
$$

In our measurement system, the temperature contribution to the refractive index is optically suppressed by a simple adjustment of the reference mirror (see Sec. II) and, therefore, only the contribution due to the concentration difference is considered in Eq. (10).

b. Fickian diffusion coefficient from a free diffusion experiment. The initial purpose of these isothermal diffusion ex- periments was to measure the Fickian diffusion coefficients, which are determined by minimizing Eq. (5). In this study, the technique is used mainly to measure the contrast factor $\eta$. The Fickian diffusion coefficient, however, can also be determined for comparison with that obtained in the thermodiffusion experiment.

\section{Experimental procedure}

The experimental procedure is divided into five steps. First, (a) the Soret cell is placed in the test section of the optical system and the mirrors of both reference beams are adjusted to obtain the infinite fringe condition in each interferogram. Then, the thermodiffusion field is initiated by $(b)$ setting a temperature difference between the upper and lower copper/liquid boundaries, which leads to a linear temperature profile within the cell in less than one minute. Next, $(c)$ the fringes obtained due to the linear temperature profile are suppressed in one of the interferometers by adjusting its reference mirror. The most important measurement of the experiment is done by $(d)$ recording the phase-shifted data until a linear concentration profile is reached within the cell, i.e., the interferogram fringes become equidistant. Finally, $(e)$ the temperature difference within the cell is set to zero to visualize the isothermal diffusion. An explanation of each step is given.

a. Placement in the optical system of the Soret cell filled with the binary solution. The Soret cell (see Fig. 2) is completely filled with a binary solution and then placed in the test section of the orthogonal phase-shifting interferometer (see Fig. 4). The cell is aligned so that the incidence of the test beams is normal to the quartz walls. After placing the cell, the real-time phase-shifting image processing is started in both interferometers. The phase-shifted data is brought to an infinite fringe condition by adjusting the mirrors of the reference beams $M 1$ and $M 4$ (see Figs. 4 and 5); it is recommended to fix the test beam mirrors $M 2$ and $M 3$ in order to guarantee the perpendicularity of the incident beams.

b. Induction of thermodiffusion within the cell. The experiment is initiated by creating a temperature difference between the upper and lower copper/ liquid boundaries. Since the Lewis number for binary liquid systems is usually in the order of $10^{2}$, the temperature field within the cell reaches a steady state much faster than the concentration field. The Lewis number for dilute aqueous aprotinin $(6.5 \mathrm{kDa})$ and lysozyme $(14.3 \mathrm{kDa})$ is about $5.6 \times 10^{2}$ and $3.2 \times 10^{2}$, respectively. Here, the thermal diffusivity of pure water at $25^{\circ} \mathrm{C}$ was taken as $1.4 \times 10^{-7} \mathrm{~m}^{2} / \mathrm{s}$ and previously reported mass diffusion coefficients ${ }^{43}$ were used to compute the Lewis number. Therefore, when applying a temperature difference of $\Delta T$ within a two-millimeter spacing, it takes only about $15 \mathrm{~s}$ for the temperature to reach a linear profile in water (within $1 \%$ of the initial temperature difference), whereas more than one hour is required for the protein molecules to reach a quasisteady state within the cell.

c. Elimination of the fringes due to the linear temperature profile. In the first couple of minutes of the experiment, the 
linear temperature profile formed within a $1-2 \mathrm{~mm}$ height cell creates a linear refractive index profile. Thermodiffusion is much slower than heat conduction and, therefore, the refractive index is almost linear in the vertical direction. Immediately after the linear temperature profile is achieved, the tilt of the mirror $M 1$ is adjusted to optically suppress the influence of the temperature on the interferogram. The inclination adjustment is done manually within $25 \mathrm{~s}$. The reference beam mirror (MI) is adjusted instead of the test beam mirror (M3) to keep the incident test beam perpendicular to the quartz glass. The thermodiffusion effect is only observed with the first interferometer (with $L 1$ as light source), i.e., the mirror $M 4$ remains fixed and unadjusted. The process of suppressing the fringes due to ${ }^{2 n} / \partial T$ is the same as that shown in Fig. 5(a), in which the mirrors $M 1$ and $M 4$ are adjusted so that the wavefronts of the test beam and reference beam become parallel to each other, i.e., the necessary condition to obtain an infinite fringe interferogram.

d. Visualization of the Soret effect-Thermodiffusion phase. The phase-shifted data from the first interferometer is visualized in an external monitor and is stored every $1 \mathrm{~s}$ for postexperimental image processing. The high-resolution visualization of the Soret effect is the main contribution of this study. This measurement continues until the fringes of the phaseshifted data (or interferogram) reach an equidistant state.

e. Visualization of Fickian diffusion - Isothermal diffusion phase. After linear concentration and temperature profiles within the cell are reached, the temperature difference be- tween the copper/liquid boundaries is brought to zero and the temperature of the cell is set to the room temperature. Here, the second interferometer (with $L 2$ as light source) is used to visualize isothermal diffusion. The recording process continues until the fringes in the phase-shifted data disappear.

After culminating the five steps $(a)-(e)$ mentioned above, the thermodiffusion and isothermal diffusion phases can be indefinitely repeated with the first and second interferometric setups, respectively, without the need of readjusting the reference mirrors while applying the same temperature difference between the upper and lower copper/liquid boundaries.

\section{RESULTS AND DISCUSSION}

\section{A. Contrast factors}

Figure 7 shows an isothermal diffusion experiment conducted with the ethanol-water binary solution. The lighter ethanol-water solution (ethanol 39.49 wt.\%) is placed over a denser solution (ethanol 37.55 wt.\%) inside a four-side transparent diffusion cell (Fine Inc. $\mathrm{W} \times \mathrm{L} \times \mathrm{H}=2 \times 10$ $\times 45 \mathrm{~mm}^{3}$ ). The diffusion process is observed with OPSI (see Fig. 4). The direction of each test beam in relation with the position of the cell is shown in Fig. 7(a); the corresponding cell dimensions and system of coordinates are also indicated. In this setup, the optical path within the diffusion cell for the test beam $L 2$ is five times larger than for $L 1$ and, therefore, the resolution for the measurement conducted with the former is five times better than that conducted with the latter. Figures 7(b) and 7(c) show the phase-shifted data obtained by using the interferometers with light source $L 1$ and $L 2$, respectively. For $L 1$, the spatial visualization range is

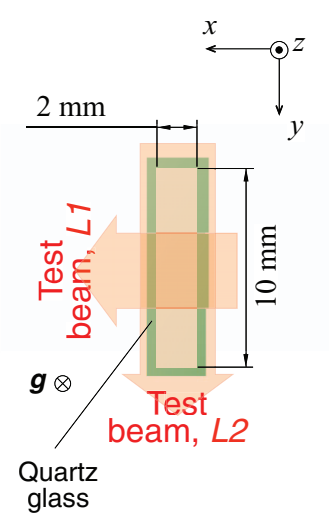

(a)

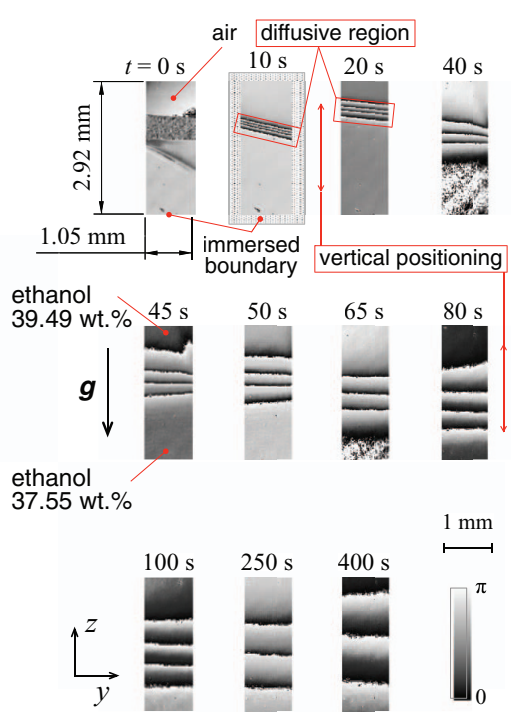

(b)

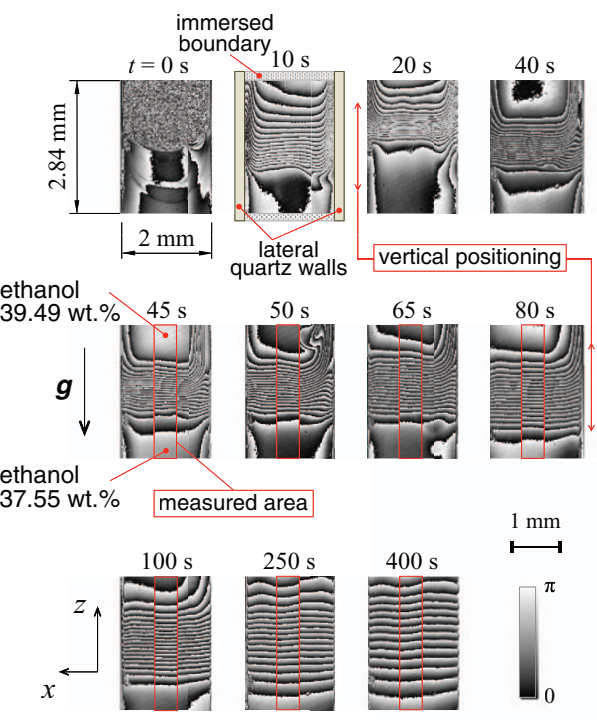

(c)

FIG. 7. Isothermal diffusion of ethanol-water solution at ethanol mean concentration $C_{m}=38.52$ wt. $\%$ and $\Delta C=1.94$ wt. $\%$. (a) The upper view of the diffusion cell with its dimensions, the direction of each test beam, and the coordinate system. (b) The diffusion process observed with the test beam $L 1$ (OP $2 \mathrm{~mm})$. (c) The diffusion process observed with the test beam $L 2(\mathrm{OP} 10 \mathrm{~mm})$. The phase difference between the upper and lower boundaries is taken after $t=45 \mathrm{~s}$. The vertical positioning of the diffusion cell is adjusted until $t=85 \mathrm{~s}$. 
$\mathrm{W} \times \mathrm{H}=1.05 \times 2.92 \mathrm{~mm}^{2}$ and the surrounding visualization boundaries are immersed surfaces. For $L 2$, the visualization range is $\mathrm{W} \times \mathrm{H}=2 \times 2.84 \mathrm{~mm}^{2}$ with its width corresponding to the length of the cell $(L=2 \mathrm{~mm})$, i.e., the lateral boundaries are impermeable walls while the upper and lower boundaries are immersed.

At $t=0 \mathrm{~s}$, the lighter solution is put in contact with the denser solution. The part of the cell that still has air is only visible with the beam $L 1$ because the meniscus at the corners of the cell blocks the beam $L 2$. At $t=10 \mathrm{~s}$, the diffusive region is thin and clearly visible with $L 1$ but not with $L 2$, where it appears thicker; this is due to the influence of the initial meniscus and the non-one-dimensionality of the diffusive field within the cell. At $t=20 \mathrm{~s}$, the vertical positioning of the cell is adjusted so that the diffusion process view is centered. The diffusion area is delimited by the lower and upper fringes, as indicated by the rectangles at $t=10 \mathrm{~s}$ and $20 \mathrm{~s}$ in Fig. 7(b). This means that the areas with the same phase shift (below the lower fringe and above the upper fringe) indicate a homogeneous concentration area and, as a consequence, there is no Fickian diffusion because $\partial C / \partial x=0$. At $t=45 \mathrm{~s}$, the diffusion field at the center is quasi-one-dimensional and, therefore, it can be used to determine the phase difference between the boundaries $(\Delta \psi)$; here $\Delta \psi_{\text {ethanol-water }}=18.74 \pi$. However, at $t=50 \mathrm{~s}$ we can see with $L 2$ a bulk of heavy solution falling near the right wall. This is due to a mistake in the injection procedure and is a cause of error in the measurement of the diffusion coefficient. In the isothermal diffusion experiments, only the data obtained by $L 2$ is used because a higher resolution is obtained with this interferometer (the number of fringes is proportional to the resolution in the concentration domain). At $t=85 \mathrm{~s}$, the vertical position of the cell is fixed, so that the phase-shifted data after $t=85 \mathrm{~s}$ can be used to determine the diffusion coefficient from the transient concentration profiles.

Figure 8 shows the optical measurements obtained from the phase-shifted data shown in Fig. 7(c). Figure 8(a) shows the phase difference profile between the test and reference beams obtained by unwrapping the phase-shifted data inside the region indicated by the rectangles after $t=45 \mathrm{~s}$, averaging at each height $z$, and shifting the curves in the phase difference domain assuming that there is an equal mass flux through both boundaries. The adjustment in the phase difference domain is necessary since the unwrapping alone produces timeunrelated phase difference profiles for each phase-shifted data, such as the one shown in Fig. 6(a). The data near the wall boundaries should be avoided until the diffusion field within the cell has reached a completely one-dimensional state. Figure 8(b) shows the phase difference between the boundaries $\Delta \psi$ as a function of time. This figure suggests that after $t=100 \mathrm{~s}$ there is a mass flux through the immersed boundaries. $\Delta \psi$ is then averaged between $t=45 \mathrm{~s}$ and $t=90 \mathrm{~s}$. In this case, $\Delta \psi_{\text {ave }}=18.74 \pi \mathrm{rad}, \Delta C_{\text {ethanol }}=1.94 \mathrm{wt} . \%$, and $\Delta l=10 \mathrm{~mm}$ and, therefore, the contrast factor defined by Eq. (9) at a mean ethanol concentration of $38.52 \mathrm{wt} . \%$ is $\eta_{\text {ethanol-water }}(38.52 \mathrm{wt} . \%)=0.97 \pi\left[\mathrm{radwt} . \%^{-1} \mathrm{~mm}^{-1}\right]$. The contrast factor has the opposite sign if the reference component in the binary system is changed. The measured

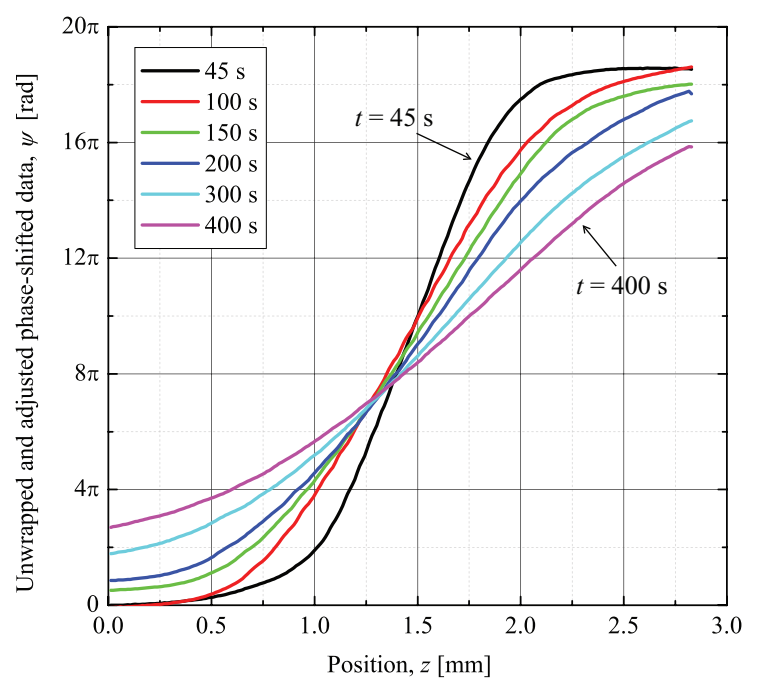

(a)

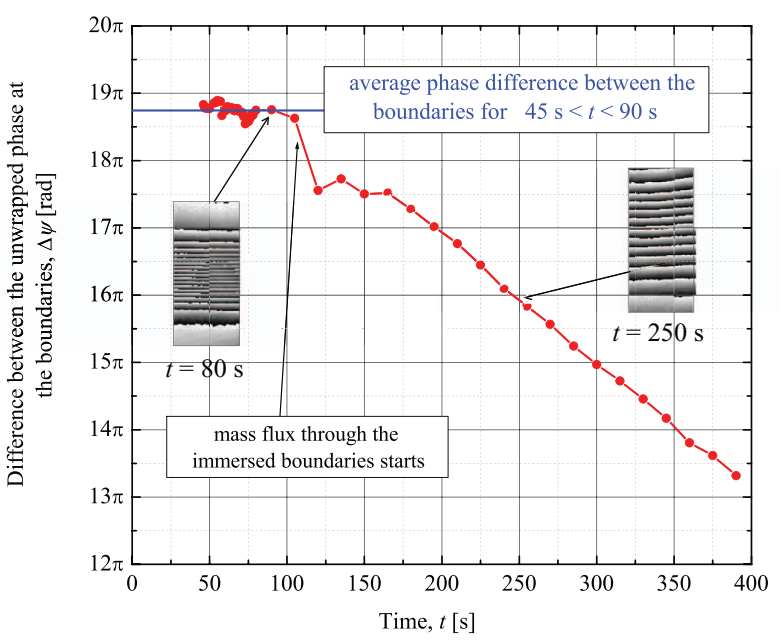

(b)

FIG. 8. Optical measurements from an isothermal diffusion experiment in an ethanol-water binary solution at ethanol mean concentration $C_{m}$ $=38.52 \mathrm{wt} . \%$ and $\Delta C=1.94 \mathrm{wt} . \%$. (a) The portion surrounded by the vertical rectangle of the phase-shifted data shown in Fig. 7(c) is unwrapped and then averaged in the $x$ direction; the resulting profiles are translated (adjusted) in the phase difference domain assuming that the mass flux through both boundaries is equal. (b) The temporal variation of $\Delta \psi$, which is the difference between the unwrapped phase at the lower and upper boundaries. $\Delta \psi$ remains constant as long as there is no mass flux through the immersed boundaries, as indicated by the phase-shifted data at $t=80 \mathrm{~s}$.

contrast factors for the four binary solutions are listed in Table II.

Figure 9 shows the isothermal diffusion field for the isobutylbenzene-dodecane binary solution. The experimental concentration profiles (gray circles) and their corresponding fittings (solid lines) are shown at intervals of $100 \mathrm{~s}$. The fitting of the numerical solution to the experimental results was conducted by inverse analysis (see Eq. (5)). The visualization range is from $z=0$ to $z=2.83 \mathrm{~mm}$, i.e., the upper and lower boundaries are immersed as indicated by the 
TABLE II. Measurement of the contrast factor $\eta$ and diffusion coefficient for the four binary solutions at mean concentration $C_{m}$. The concentration in the contrast factors is given for the denser components, which are underlined for each solution. The reference values for ethanol-water (water 60.13 wt.\%) and isobutylbenzene-dodecane (IBB-C12 $50 \mathrm{wt} . \%$ ) are included. The factor $\partial n / \partial C$ is not included for the protein aqueous solutions to avoid confusion due to the different units used in the concentration term.

\begin{tabular}{|c|c|c|c|c|c|c|}
\hline Binary solution & Concentration, $C_{m}$ & Contrast factor, $\eta$ & $\begin{array}{c}\text { Measured } \\
\left(\frac{\partial n}{\partial C}\right)_{p, T} \times 10^{-2}\end{array}$ & $\begin{array}{c}\text { Reference } \\
\left(\frac{\partial n}{\partial C}\right)_{p, T} \times 10^{-2}\end{array}$ & $\begin{array}{c}\text { Measured } \\
D\left(\mathrm{~m}^{2} / \mathrm{s} \times 10^{-10}\right)\end{array}$ & $\begin{array}{c}\text { Reference } \\
D\left(\mathrm{~m}^{2} / \mathrm{s} \times 10^{-10}\right)\end{array}$ \\
\hline Ethanol-water & 61.48 wt. $\%$ & $-0.97 \pi(\mathrm{wt} . \% \mathrm{~mm})^{-1}$ & -3.07 & -3.55 (Ref. 13) & $4.31 \pm 0.92$ & $4.32($ Ref. 11) \\
\hline IBB-C12 & 50.0 wt. $\%$ & $1.93 \pi(\text { wt. } \% \mathrm{~mm})^{-1}$ & 6.01 & $6.28 \pm 0.04($ Ref. 13$)$ & $9.57 \pm 0.24$ & $9.50 \pm 0.4($ Ref. 12$)$ \\
\hline Aprotinin-water & $1.0 \mathrm{mg} / \mathrm{ml}$ & $0.29 \pi(\mathrm{g} / \mathrm{l} \mathrm{mm})^{-1}$ & $\ldots$ & $\ldots$ & $2.54 \pm 0.14($ Ref. 43$)$ & $\ldots$ \\
\hline$\overline{\text { Lysozyme-water }}$ & $1.0 \mathrm{mg} / \mathrm{ml}$ & $-0.51 \pi(\mathrm{g} / \mathrm{l} \mathrm{mm})^{-1}$ & $\ldots$ & $\ldots$ & $4.48 \pm 0.18($ Ref. 43$)$ & $\ldots$ \\
\hline
\end{tabular}

concentration gradients at the boundaries. The phase-shifted data at $t=0 \mathrm{~s}, 100 \mathrm{~s}$, and $500 \mathrm{~s}$ are shown in the insets. The diffusion coefficient obtained at $t=500 \mathrm{~s}$ is $D=9.56 \times 10^{-10}$ $\mathrm{m}^{2} / \mathrm{s}$. Figure 10 shows the transient concentration profiles and the phase-shifted data at $t=100 \mathrm{~s}$ and $t=500 \mathrm{~s}$ obtained for the lysozyme-water binary solutions at $C_{m}=1 \mathrm{mg} / \mathrm{ml}$. As in the case of the reference mixtures, the transient concentration profiles of protein aqueous solutions can be observed.

The isothermal diffusion coefficient obtained by averaging the determined $D$ at each fit is listed in Table II with its corresponding standard deviation for the four binary solutions: ethanol-water (water $61.48 \mathrm{wt} . \%$ ), isobutylbenzenedodecane $(50.0$ wt. $\%)$, aprotinin-water $(1.0 \mathrm{mg} / \mathrm{ml})$, and lysozyme-water $(1.0 \mathrm{mg} / \mathrm{ml})$. The reference value of the diffusion coefficient for non-protein solutions is included. A good agreement with the literature was found for the reference solutions. However, the standard deviation for the diffusion co-

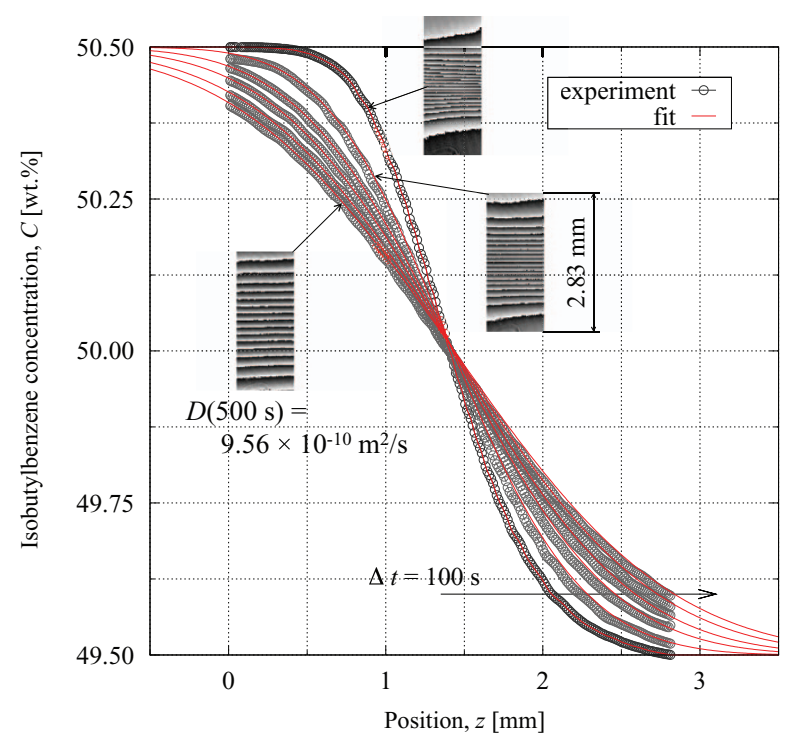

FIG. 9. Transient concentration profiles within the diffusion cell for the isobutylbenzene-dodecane binary solution. The experimental and numerical profiles are indicated by gray circles and solid lines, respectively. The visualization range is from $z=0$ to $z=2.83 \mathrm{~mm}$. Each numerical profile, which is based on Fick's law assuming a constant diffusion coefficient, is fitted to the experimental results by inverse analysis. In the numerical calculations, the first experimental profile is used as initial condition. The phase-shifted data for $t=0,100$, and $500 \mathrm{~s}$ is shown in the insets. The diffusion coefficient at $t$ $=500 \mathrm{~s}$ is $D=9.56 \times 10^{-10} \mathrm{~m}^{2} / \mathrm{s}$. The average diffusion coefficient for all fits and its corresponding standard deviation is shown in Table II. efficient of the ethanol-water mixture is quite high, about $20 \%$ of its value; the disturbance observed at $t=50 \mathrm{~s}$, which is shown in Fig. 7(c), must have influenced the accuracy of the measurement of the diffusion coefficient. The main goal of this experiment is to measure the contrast factor, so reconducting the experiment only for diffusion measurements is unnecessary.

\section{B. Benchmark mixtures}

Thermodiffusion experiments were conducted with two well-studied binary mixtures, i.e., ethanol-water and isobutylbenzene-dodecane, in order to validate the proposed method. Figure 11 shows the phase-shifted data obtained in a thermodiffusion experiment for an ethanol-water solution at an initial homogeneous concentration of $39.12 \mathrm{wt} . \%$ for the ethanol component (water 60.88 wt.\%). Two phase-shifted data are simultaneously obtained by OPSI (see Fig. 4). Here, the OP within the cell for $L 1$ and $L 2$ is $20 \mathrm{~mm}$ and $10 \mathrm{~mm}$, respectively, just as in Fig. 2(c). Therefore, the resolution of the concentration measurement with $L 1$ is double of that obtained with $L 2$.

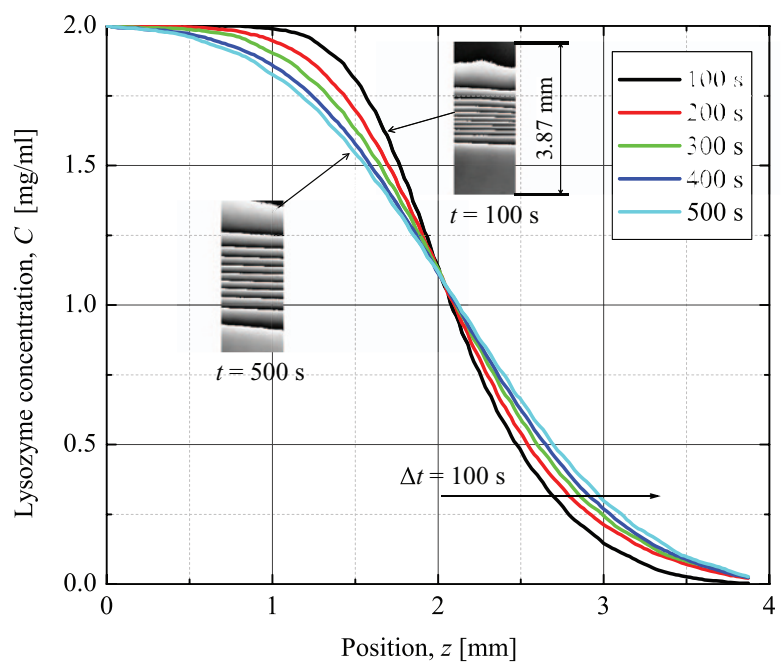

FIG. 10. Experimental transient concentration profiles of the protein aqueous solutions of lysozyme-water at $C_{m}=1 \mathrm{mg} / \mathrm{ml}$. The OP is $20 \mathrm{~mm}$. The phase-shifted data (modulo $2 \pi$ ) corresponding to the initial and final concentration profiles are shown in the insets. The diffusion process starts at $t=0 \mathrm{~s}$. 


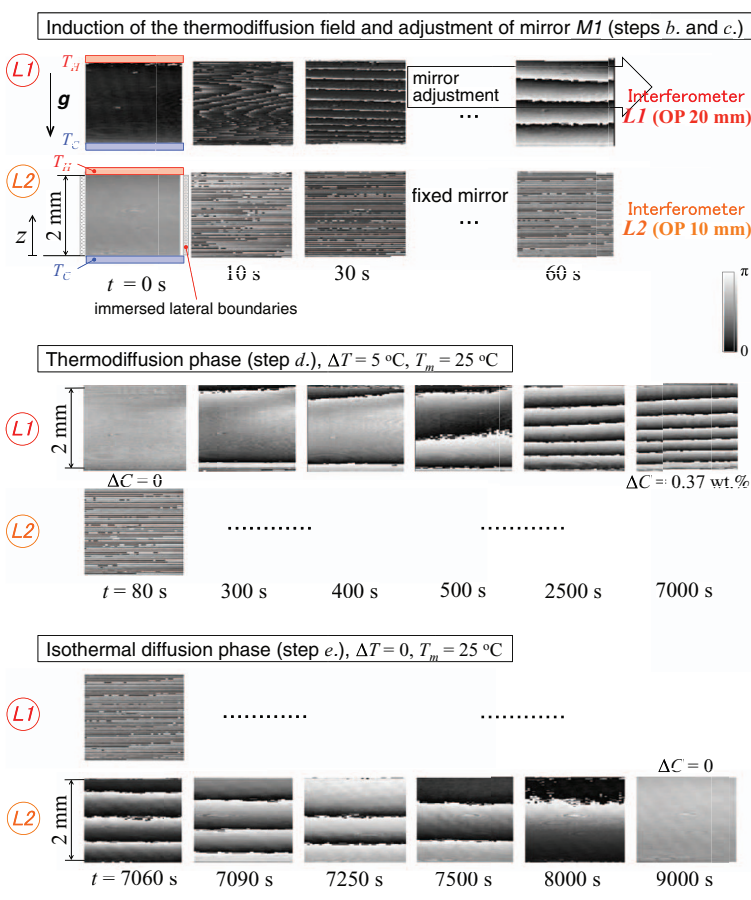

FIG. 11. Phase-shifting interferometric measurements of the thermodiffusion and isothermal diffusion fields of the ethanol-water binary solution (ethanol 39.12 wt.\%) inside the Soret cell. The views from the orthogonally aligned interferometers with light sources $L 1$ (OP $20 \mathrm{~mm}$ ) and $L 2$ $(\mathrm{OP} 10 \mathrm{~mm})$ are shown at different experimental times. The entire height of the cell, i.e., $2 \mathrm{~mm}$, is recorded by the phase-shifted data. The induction of the thermodiffusion field is conducted by applying a temperature difference of $\Delta T=5{ }^{\circ} \mathrm{C}\left(T_{m}=25^{\circ} \mathrm{C}\right)$ and adjusting the tilt of the mirror $M 1$. The temperature reaches a linear profile between the copper blocks within $75 \mathrm{~s}$. The mirror adjustment is finalized at $80 \mathrm{~s}$, which is the time at which the measurement of the thermodiffusion phase is initialized. At $t=7000 \mathrm{~s}$, the ethanol concentration profile has reached a quasi-steady state. At $t=7001 \mathrm{~s}$, the binary solution is set to an isothermal state $\left(T=25^{\circ} \mathrm{C}\right)$ and the Fickian diffusion process is then visualized with the test beam $L 2$ until the binary solution becomes homogeneous.

The ethanol-water binary solution is injected inside the Soret cell, which is then placed in the test section of the OPSI system. As shown in Fig. 11, the thermodiffusion field is induced by applying a temperature difference of $\Delta T=5^{\circ} \mathrm{C}$ (mean temperature $T_{m}=25^{\circ} \mathrm{C}$ ) with the temperature control system previously described; $T_{\text {room }}$ is $25^{\circ} \mathrm{C}$ throughout the course of the experiment. At $t=50 \mathrm{~s}$ the temperature at the upper and lower boundaries has reached a constant value. After reaching a linear temperature profile between these boundaries, as indicated by the infinite fringe condition of $L I$ at $t=80 \mathrm{~s}$, there is a constant thermodiffusive mass flux throughout the binary solution. This infinite fringe condition at the beginning of the thermodiffusion phase is achieved by manually adjusting the tilt of the mirror $M 1$. In contrast, the fringe pattern obtained with $L 2$ is too dense to subtract an unwrappable phase-shifted data with the CCD camera of pixel resolution $640 \times 480$. Hence, the thermodiffusion measurement is conducted only with $L 1$, as shown in Fig. 11. Then, fringes start appearing near the upper and lower boundaries due to thermophoretic induced con- centration gradients, as shown in the phase-shifted data at $t=300 \mathrm{~s}$ and $t=400 \mathrm{~s}$. It is only in the region where fringes appear that mass transport due to Fickian diffusion occurs and opposes thermodiffusion, as described by Eq. (2). At this point, we can know if the species are thermophobic $\left(S_{T}>0\right)$ or thermophilic $\left(S_{T}<0\right)$ by comparing the phase-shifted data obtained with $L 1$ (thermodiffusion phase in Fig. 11) and the phase-shifted data obtained in the preliminary experiments (snapshots in Fig. 7). We can see from the preliminary experimental results that the intensity gradient between two fringes in the phase-shifted data is positive (black to white isovalues) when moving upwards (increasing $z$ values); in these experiments the denser solution (which is richer in water) is placed below. Since we also have a positive gradient between two fringes in the thermodiffusion phase shown in Fig. 11, we can conclude that in this thermodiffusion experiment the water molecules migrate to the lower cold section of the cell. Therefore, the water molecules in this ethanolwater solution are thermophobic $\left(S_{T}>0\right)$. After $t=400 \mathrm{~s}$ in the thermodiffusion phase, fringes progressively occupy the whole height of the cavity until the concentration field reaches a quasi-steady linear state, as indicated by the seemingly equidistant fringes in the phase-shifted data of $L 1$ at $t=7000 \mathrm{~s}$. These equidistant fringes are then connected to determine the phase difference $\Delta \psi$ between the upper and lower boundaries. At $t=7000 \mathrm{~s}, \Delta \psi$ becomes $7.08 \pi$, and by substituting $\Delta \psi, \eta_{\text {ethanol-water }}$ (obtained in the preliminary experiments), and $\Delta l=20 \mathrm{~mm}$ in Eq. (9), the concentration difference between the upper and lower boundaries is found to be $\Delta C=0.37 \mathrm{wt} . \%$.

At $t=7001 \mathrm{~s}$, the temperature control system is used to induce the isothermal phase $\left(T=25^{\circ} \mathrm{C}\right)$ and Fickian diffusion is then visualized with the beam $L 2$. During this temperature control process, which takes about $70 \mathrm{~s}$, there is a range $(7008 \mathrm{~s}<t<7028 \mathrm{~s})$ where the temperature at the lower boundary is larger than that at the upper boundary. This might result in hydrodynamic instabilities due to $R a>0$. In this situation, the Rayleigh number $R a=726$ is estimated by substituting in Eq. (4) the following: $\Delta T=2{ }^{\circ} \mathrm{C}$ (more than the maximum $\Delta T$ in this range), $l=0.002 \mathrm{~m}, g=9.8 \mathrm{~m} / \mathrm{s}^{2}$, and the corresponding thermophysical properties of the ethanol-water solution $\beta=5.94 \times 10^{-4} 1 / \mathrm{K}, v=1.08$ $\times 10^{-6} \mathrm{~m}^{2} / \mathrm{s}, \alpha=1.18 \times 10^{-7} \mathrm{~m}^{2} / \mathrm{s}$. The resulting $R a$ is less than half of the critical Rayleigh number $R a_{c}=1732.5$, which was calculated for this geometry by a continuation method previously described ${ }^{53}$ (assuming $\Delta C=0$ ). Therefore, we can guarantee that there are no disturbances due to natural convection. In fact, the actual critical Rayleigh number should be larger than the calculated $R a_{c}$, because the concentration gradients present in the cell, which were induced by the original temperature gradient of the thermodiffusion phase, are stabilizing. After the temperature has reached a constant value within the cell, quasi-equidistant fringes are observed with $L 2$ at $t=7060 \mathrm{~s}$. This indicates that there is a linear concentration profile at the beginning of the isothermal diffusion phase. Moreover, when comparing the number of fringes obtained in the phase-shifted data of $L 1$ and $L 2$ at $t=7000 \mathrm{~s}$ and $t=7060 \mathrm{~s}$, respectively, (i.e., 6 fringes in the former case and 3 fringes in the latter case) it is evident that the resolution of 
(a)

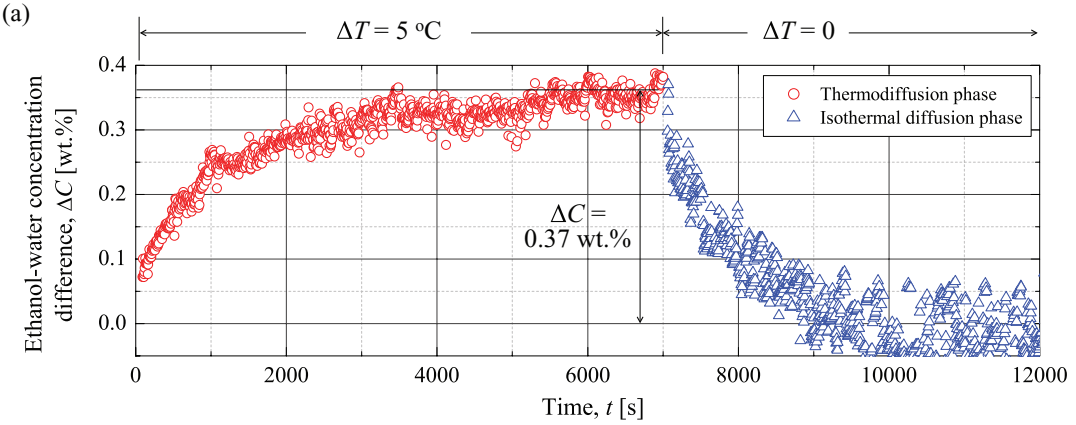

(b)

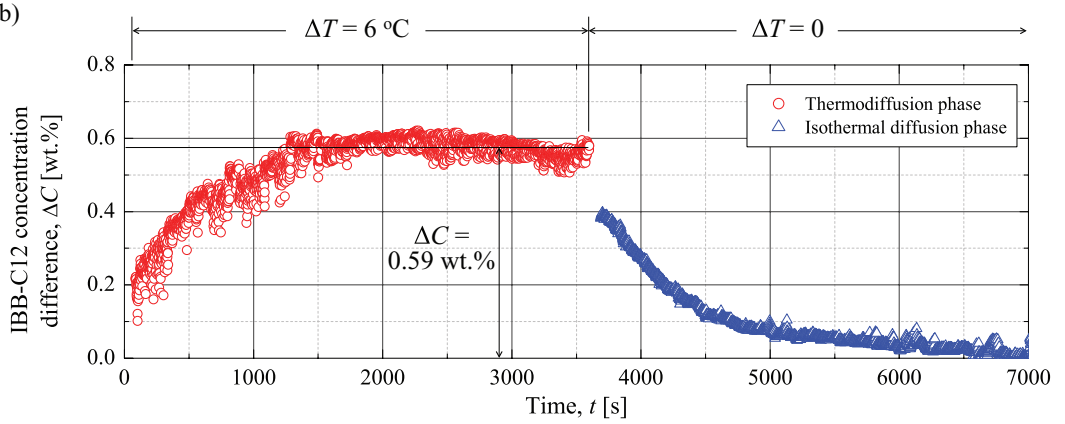

FIG. 12. Temporal variation of the concentration difference $\Delta C$ between the upper and lower walls for (a) ethanol in the ethanol-water solution, and (b) dodecane in the isobutylbenzene-dodecane (IBB-C12) solution. The lightest component in each liquid pair, i.e., (a) ethanol and (b) dodecane, is taken as reference for the concentration difference. The total experimental time is $12000 \mathrm{~s}$ for the former case and $7000 \mathrm{~s}$ for the latter case. Some snapshots of the phase-shifted data for the ethanol-water experiment are shown in Fig. 11. The temperature difference between the upper and lower boundaries in the thermodiffusion phase of ethanol-water and isobutylbenzene-dodecane is $\Delta T=5{ }^{\circ} \mathrm{C}$ and $\Delta T=6{ }^{\circ} \mathrm{C}$, respectively. The OP in the measurement shown in (a) is $20 \mathrm{~mm}$ for the thermodiffusion phase and $10 \mathrm{~mm}$ for the isothermal diffusion phase. The OP in the measurement shown in (b) is $10 \mathrm{~mm}$ for the thermodiffusion phase and $20 \mathrm{~mm}$ for the isothermal diffusion phase. The scattering for the $\Delta C$ points is larger for the short OP measurements.

the measurement with $L 1$ is double of that with $L 2$ due to the longer OP. The isothermal diffusion phase is then visualized until the binary solution becomes homogeneous, as indicated by the homogenous intensity (infinite fringe condition) of the phase-shifted data at $t=9000 \mathrm{~s}$ in Fig. 11.

Figure 12 shows the temporal variation of the concentration difference $\Delta C$ between the upper and lower walls for the ethanol-water (Fig. 12(a)) and isobutylbenzene-dodecane (Fig. 12(b)) binary solutions. $\Delta C$ in Fig. 12(a) corresponds to that of ethanol, while $\Delta C$ in Fig. 12(b) corresponds to that of dodecane. Since ethanol and dodecane are the lightest components in each liquid pair, the hydrodynamic stability of the solution increases with time during the thermodiffusion phase. Here, the contrast factor $\eta$ for ethanolwater at $C_{m}=38.5 \mathrm{wt} . \%$ and isobutylbenzene-dodecane at $C_{m}=50 \mathrm{wt} . \%$ (see Table II) was used to determine $\Delta C$. We assume that the contrast factor for the ethanol-water mixture between $C_{m}=38.5$ wt. $\%$ and $C_{m}=39.12 \mathrm{wt} . \%$ does not vary significantly. ${ }^{54}$ Moreover, in the ethanol-water experiment, the OP for the thermodiffusion and isothermal diffusion phases was $20 \mathrm{~mm}$ and $10 \mathrm{~mm}$, respectively, i.e., the concentration resolution in the thermodiffusion measurement is two times larger than in the isothermal diffusion measurement. This explains a larger scattering of the concentration difference $\Delta C$ points in the isothermal diffusion phase. In contrast, in the isobutylbenzene-dodecane experiment, the OP for the thermodiffusion and isothermal diffusion phases was $10 \mathrm{~mm}$ and $20 \mathrm{~mm}$, respectively, values opposite to the ones chosen in the ethanol-water experiment, and the scattering of $\Delta C$ is now larger in the thermodiffusion phase. These results show that the measurements with a shorter OP result in a larger scattering of $\Delta C$.

The measurements shown in Fig. 12 are similar to those conducted with OBD technique where the measurement is based on the temporal variation of a local refractive index within the cell. Nevertheless, the advantages of phase-shifting interferometry become evident when analyzing the phaseshifted data in more depth. Figure 13 shows the transient concentration profiles for the ethanol-water solution (ethanol 39.12 wt.\%) obtained from the phase-shifted data of the thermodiffusion phase; the phase-shifted data at $t=100 \mathrm{~s}, 600 \mathrm{~s}$ and $7000 \mathrm{~s}$ are also shown as insets (see more snapshots in Fig. 11). The thermophilic nature of the ethanol molecules in this liquid pair is evident. Moreover, we can confirm that the concentration profile within the cell in its steady state is quasi-linear. This final state corresponds to the state where the Fickian diffusion and thermodiffusion, whose rates are written in Eq. (2), balance each other. We can also see in Fig. 13 that there is some waving of the concentration profiles, particularly near $z=0$, i.e., close to the lower cold boundary. This is due to the beam deflection problem shown in Fig. 14. This problem is worsen when the OP within the cell or the temperature difference between the walls $\Delta T$ is larger, making measurements with very long OP or large $\Delta T$ quite problematic. In order to reduce the beam deflection problem for the beams which are not reflected by the lower copper wall, the 


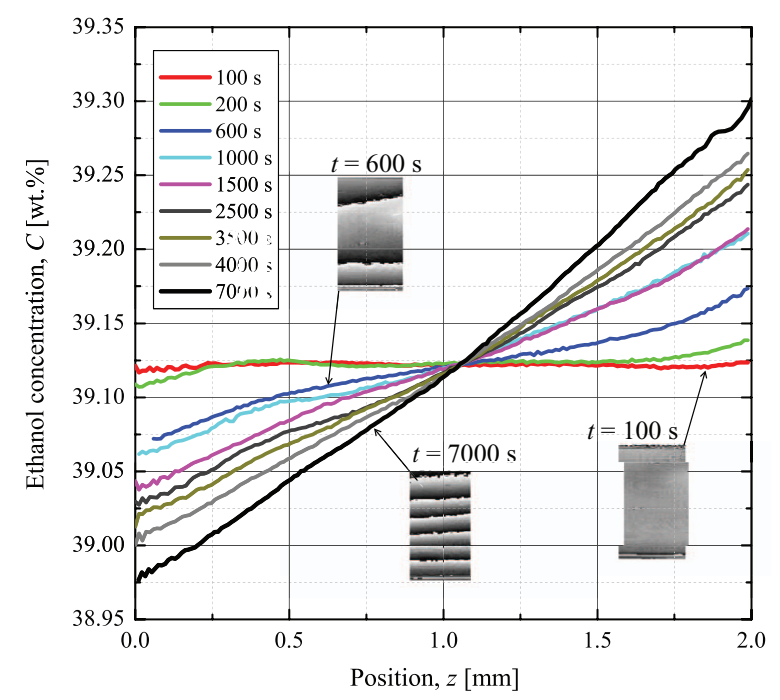

FIG. 13. Transient concentration profiles for the ethanol component in the ethanol-water binary solution during its thermodiffusion phase. Thermodiffusion drives the ethanol molecules to the upper region, i.e., the high temperature region, and the water molecules to the lower region, i.e., the low temperature region. Therefore, the ethanol and water molecules have thermophilic and thermophobic behavior, respectively.

focus point of the test beam was set to match the inner plane of the glass wall that is nearest to the CCD camera, as suggested by Mialdun and Shevtsova, ${ }^{19}$ for each test beam $L 1$ and $L 2$. This improved the quality of the measurement during the thermodiffusion phase.

Figure 15 shows the transient concentration profiles during the isothermal diffusion phase of the isobutylbenzenedodecane binary solution $(50 \mathrm{wt} \%)$; the insets show the phase-shifted data at $t=3700 \mathrm{~s}$ and $6100 \mathrm{~s}$. This measurement indicates that, after the thermodiffusion phase, the isobutylbenzene molecules have accumulated in the lower cold part of the cell, revealing the thermophobic nature of the isobutyl-

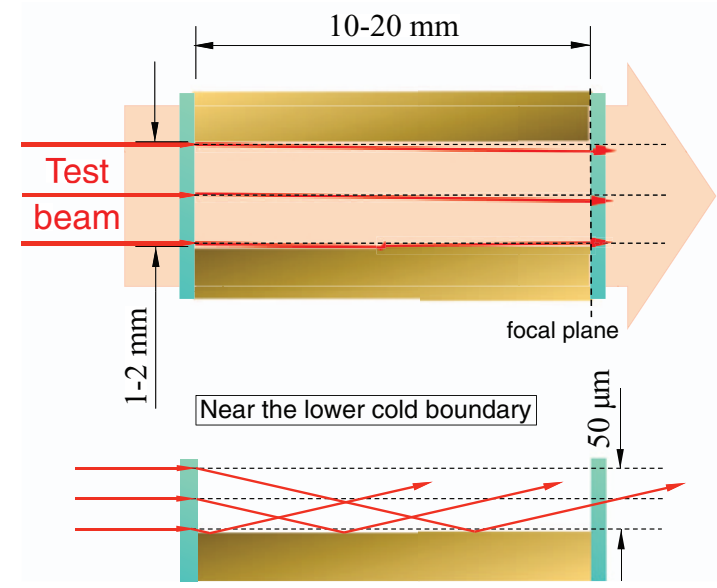

FIG. 14. Beam deflection problem through the Soret cell with focus on the lower cold boundary (for $R a<0$ ). The cold region inside the solution has a higher refractive index $n$ than the upper hot region. Therefore, the beam is curved towards the bottom of the cell.

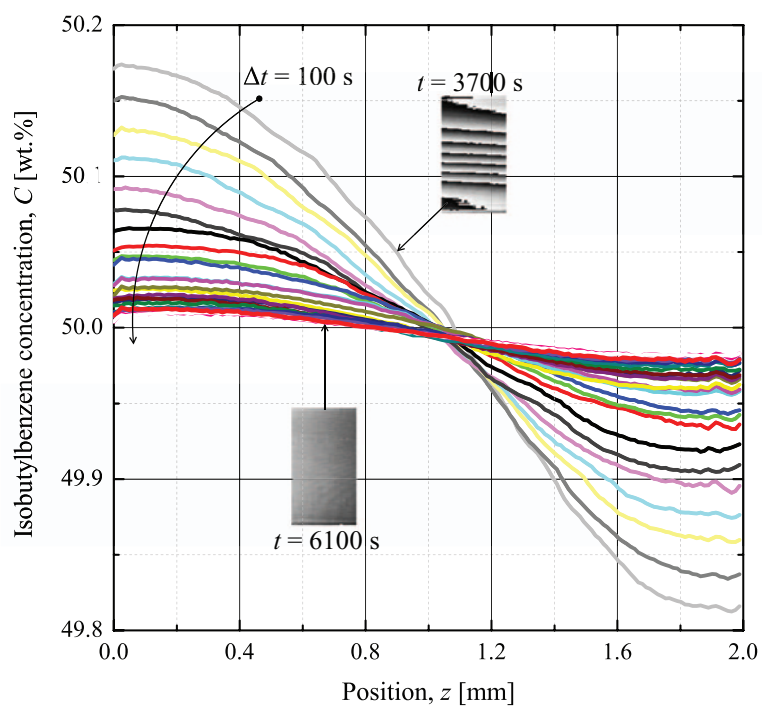

FIG. 15. Transient concentration profiles during the isothermal diffusion phase of the isobutylbenzene- dodecane binary solution (50 wt.\%). The profiles are shown at times separated by $\Delta t=100 \mathrm{~s}$ starting at $t=3700 \mathrm{~s}$. After the thermodiffusion phase, the isobutylbenzene molecules have accumulated in the lower cold part of the cell, i.e., the isobutylbenzene molecules are thermophobic. The phase-shifted data at two different times is shown in the insets.

benzene molecules. We can also observe that the concentration gradient at the copper/liquid boundaries is zero, in accordance to the impermeable condition $\left(J_{C}=0\right)$ at the wall with $\Delta T=0$. In contrast, in a thermodiffusion phase, a concentration gradient is found in the vicinity of the wall (see Fig. 13) accounting for the equilibrium between Fickian diffusion and thermodiffusion in an unchanged $J_{C}=0$ condition. Furthermore, the transient concentration profiles in the isothermal phase can be taken as $C_{\text {exp }}$ in Eq. (5) in order to minimize the objective function $\delta$ and yield the isothermal diffusion coefficient of the liquid pair at $T_{m}$. Hence, thermodiffusion can be used as a mean to create an initial concentration condition for an isothermal diffusion experiment. This is a particular advantage for solutions that are too viscous to handle by using more conventional methods. ${ }^{41,42,55}$ Moreover, the beam deflection problem described in Fig. 14 is much less evident during the isothermal diffusion phase $\left({ }^{2} / \partial T=0\right)$ than during the thermodiffusion phase. This occurs because, for the concentration and temperature differences managed in these experiments, the influence of the temperature gradient on the refractive index $(\partial n / \partial T)$, which is indicated by the dense fringes pattern of $L 2$ at $t=60 \mathrm{~s}$ in Fig. 11, is much larger than the influence of the concentration gradient on the refractive index $(\partial n / \partial C)$, which is indicated by the few fringes observed in the isothermal diffusion phase with $L 2$ at $t=7060 \mathrm{~s}$ in Fig. 11.

Table III shows the Soret coefficients (from Eq. (3)), isothermal diffusion coefficients (by optimizing Eq. (5)), and thermodiffusion coefficients (from Eq. (1)) obtained in this study by using OPSI for the two benchmark solutions. The reference values are also listed. A good agreement with the 
TABLE III. The Soret coefficient $S_{T}$, the isothermal diffusion coefficient $D$, and the thermodiffusion coefficient $D_{T}$ for the two benchmark binary solutions measured in the present study at a mean concentration $C_{m}$. The reference component is underlined. Literature values are written in parentheses for comparison.

\begin{tabular}{lcccc}
\hline \hline Liquid pair & $C_{m}($ wt.\% $)$ & $S_{T}\left(\mathrm{~K}^{-1} \times 10^{-3}\right)$ & $D\left(\mathrm{~m}^{2} / \mathrm{s} \times 10^{-10}\right)$ & $D_{T}\left(\mathrm{~m}^{2} \mathrm{~K}^{-1} / \mathrm{s} \times 10^{-12}\right)$ \\
\hline Ethanol-water & 60.88 & $3.09(3.23$, Ref. 11) & 4.31 & $1.33(1.37$, Ref. 11) \\
IBB-C12 & 50.0 & $3.86(3.9 \pm 0.1$, Ref. 12) & 9.57 & $3.69(3.7 \pm 0.2$, Ref. 12) \\
\hline \hline
\end{tabular}

literature was obtained, validating the experimental technique and the analysis method proposed in this study.

\section{Protein thermodiffusion}

Thermodiffusion in protein aqueous solutions (thermophoresis) is discussed as an application of the measurement technique proposed in this study. The purpose of this section is to verify whether thermophoresis of macrobiomolecules can be observed and measured with OPSI. Therefore, no extra measures are taken to fix the ionic strength of the solution or its $\mathrm{pH}$ level, i.e., the protein is used as solute without any further purification and dissolved in distilled water at $25^{\circ} \mathrm{C}$. Figure 16 shows the phase-shifted data obtained during two thermodiffusion experiments for a dilute aprotinin-water $(3 \mathrm{mg} / \mathrm{ml})$ binary solution: at $\Delta T=5^{\circ} \mathrm{C}$,
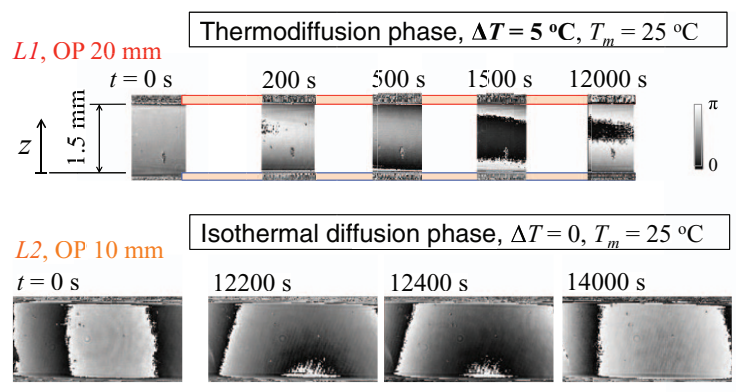

(a)

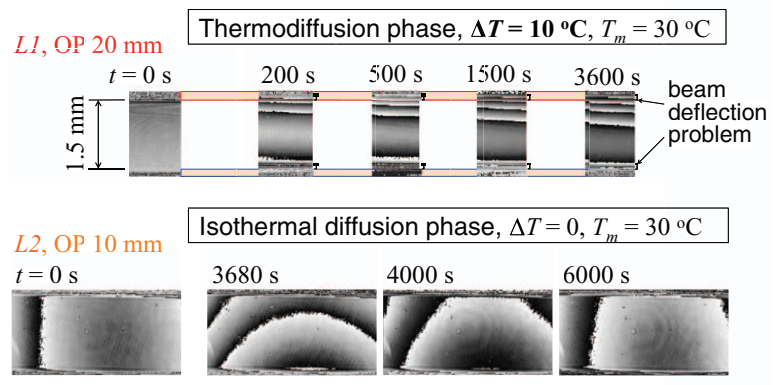

(b)

FIG. 16. Phase-shifted data for two thermodiffusion experiments of a dilute aprotinin-water $(3 \mathrm{mg} / \mathrm{ml})$ binary solution inside a Soret cell of $h=1.5 \mathrm{~mm}$. In the first experimental run (a) $\Delta T=5^{\circ} \mathrm{C}, T_{m}=25^{\circ} \mathrm{C}$, in the second experimental run (b) $\Delta T=10^{\circ} \mathrm{C}, T_{m}=30^{\circ} \mathrm{C}$. The fringes observed at $t=0 \mathrm{~s}$ are due to optical defects of the quartz walls. The fringes due to thermophoresis are clearly observed in both the thermodiffusion and isothermal diffusion phases. The beam deflection problem at $\Delta T=10^{\circ} \mathrm{C}$ and $\mathrm{OP}$ $=20 \mathrm{~mm}$ becomes more prominent.
$T_{m}=25^{\circ} \mathrm{C}$, and $\Delta T=10^{\circ} \mathrm{C}, T_{m}=30^{\circ} \mathrm{C}$. The height of the cell was shortened to $h=1.5 \mathrm{~mm}$ in order to reduce the measurement time, which is expected to be longer for protein molecules, such as aprotinin (see Sec. III A 1 b). The visualization width for $L 1$ is shorter than for $L 2$. Optical defects prevent us from achieving the infinite fringe condition with $L 2$ at $t=0 \mathrm{~s}$, even though the solution is in a homogenous state (i.e., $\Delta T=0$ and $\Delta C=0$ ). Finding the deformed wavefront, as described in Fig. 5(b), and then subtracting it to the unwrapped data at time $t$ can correct this optical problem.

The first experimental run at $\Delta T=5^{\circ} \mathrm{C}$, which is shown in Fig. 16(a), was conducted in accordance with the temperature ranges chosen in the validation experiments (see Fig. 11). However, very few fringes were observed in the steady state at $t=12000 \mathrm{~s}$ and at the beginning of the isothermal diffusion phase at $t=12200 \mathrm{~s}$. As discussed in Sec. IV B, the number of fringes is proportional to the resolution of the concentration measurement. Therefore, a second experimental run, which is shown in Fig. 16(b), was conducted at a higher temperature difference, $\Delta T=10^{\circ} \mathrm{C}$, in an attempt to increase the resolution of the measurement by obtaining more fringes in the phase-shifted data. The mean temperature within the cell and room temperature were set to $T_{m}=$ $30^{\circ} \mathrm{C}$ and $T_{\text {room }}=27^{\circ} \mathrm{C}$, respectively; these temperature values enable us to use more effectively the surrounding air as heat sink for the Peltier modules. As shown in Fig. 16(b), the thermophoretic-induced concentration gradients inside the protein aqueous solution are clearly observed at the end of the thermodiffusion phase at $t=3600 \mathrm{~s}$ and the beginning of the isothermal diffusion phase at $t=3680 \mathrm{~s}$. However, the high temperature difference $\Delta T$ between the walls increases the beam deflection problem described in Fig. 14. This jeopardizes the ability to measure the Soret coefficient only from the thermodiffusion phase since the exact concentration value at the boundaries becomes unknown. On the other hand, the isothermal data does not have the beam deflection problem and, thus, they can be used to determine the thermodiffusion and isothermal diffusion coefficients.

At this point, we can determine whether the aprotinin molecules $\left(6.5 \mathrm{kDa}\right.$ ) dissolved in water (at $C_{m}=3 \mathrm{mg} / \mathrm{ml}$ ) are thermophobic or thermophilic by observing the phase-shifted data in the isothermal phase shown in Fig. 16. In the preliminary experiments, we observed that phase-shift $\varphi$ increases with increasing concentration of aprotinin molecules (positive contrast factor $\eta$ ). In the isothermal phase, on the other hand, we observe that the phase-shift $\varphi$ increases with the position $z$ from the lower wall, indicating that the concentration increases with $z$ and, thus, we can infer that the aprotinin molecules have moved to the upper heated wall during the thermodiffusion phase. Therefore, the aprotinin molecules in 


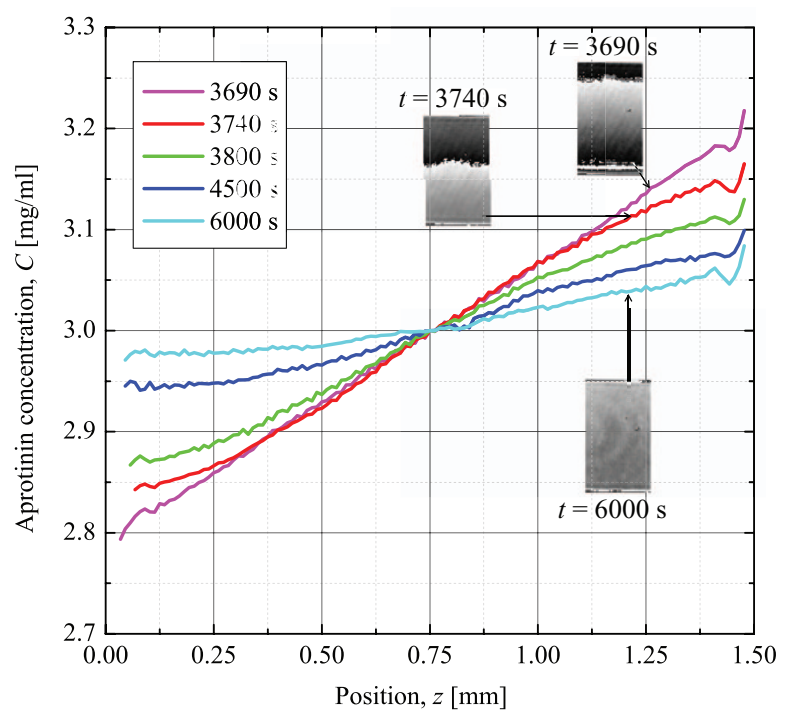

FIG. 17. Transient concentration profile during the isothermal diffusion phase of the second experimental run of the aprotinin-water binary solution. The height of the cell is $h=1.5 \mathrm{~mm}$, and the temperature difference applied during the thermodiffusion phase is $\Delta T=10^{\circ} \mathrm{C}$. Three phase-shifted data at $t=3690 \mathrm{~s}, 3740 \mathrm{~s}$, and $6000 \mathrm{~s}$ are shown in the insets. The aprotinin molecules exhibit a thermophilic behavior $\left(S_{T}<0\right)$ within the aqueous surrounding.

pure water $\left(C_{m}=3 \mathrm{mg} / \mathrm{ml}\right)$ are thermophilic, i.e., the Soret coefficient $S_{T}$ is negative for this species.

Figure 17 shows the transient concentration profiles in the isothermal diffusion phase for the second experimental run of the aprotinin-water binary solution (see Fig. 16(b)). Here, the contrast factor $\eta$ for aprotinin-water at $C_{m}=1$ $\mathrm{mg} / \mathrm{ml}$ and at $T=25^{\circ} \mathrm{C}$ (see Table II) was used to determine the concentration profiles at $C_{m}=3 \mathrm{mg} / \mathrm{ml}$, i.e., we assume that the contrast factor does not change significantly within these temperature and concentration ranges. The thermophilic nature of the aprotinin molecules is evident due to a higher concentration of molecules that have accumulated near the upper hot boundary after the thermodiffusion phase. Furthermore, a quasi-linear profile is detected at the beginning of the isothermal diffusion phase at $t=3690 \mathrm{~s}$. In contrast, a linear profile was not observed at the beginning of the isothermal phase for the ethanol-water solution (see Fig. 15) due to the higher diffusion coefficient of this liquid pair. We can thus use the linear profile at $t=3690 \mathrm{~s}$, shown in Fig. 17, to estimate the concentration difference $\Delta C$ between the plates obtained at the end of the thermodiffusion phase. We obtain $\Delta C$ $=0.4 \mathrm{mg} / \mathrm{ml}$ for this experiment where $C_{0}=3 \mathrm{mg} / \mathrm{ml}$ and $\Delta T=10^{\circ} \mathrm{C}$. In order to obtain the Soret coefficient from Eq. (3), we first need to change the units of concentration from $[\mathrm{mg} / \mathrm{ml}]$ to $[\mathrm{wt} . \%]$. This is done by assuming that the density of water at $T_{m}=30{ }^{\circ} \mathrm{C}$ is $\rho=0.9956 \mathrm{~g} / \mathrm{ml}$. Then, the mean concentration and concentration difference become $C_{0}$ $=0.3 \mathrm{wt} . \%$ and $\Delta C=0.04 \mathrm{wt} . \%$. Substituting these values in Eq. (3) yields the negative Soret coefficient $S_{T}=-1.34$ $\times 10^{-2} \mathrm{~K}^{-1}$.

Figure 18 shows some concentration profiles for the thermodiffusion phase of a lysozyme-water binary solution

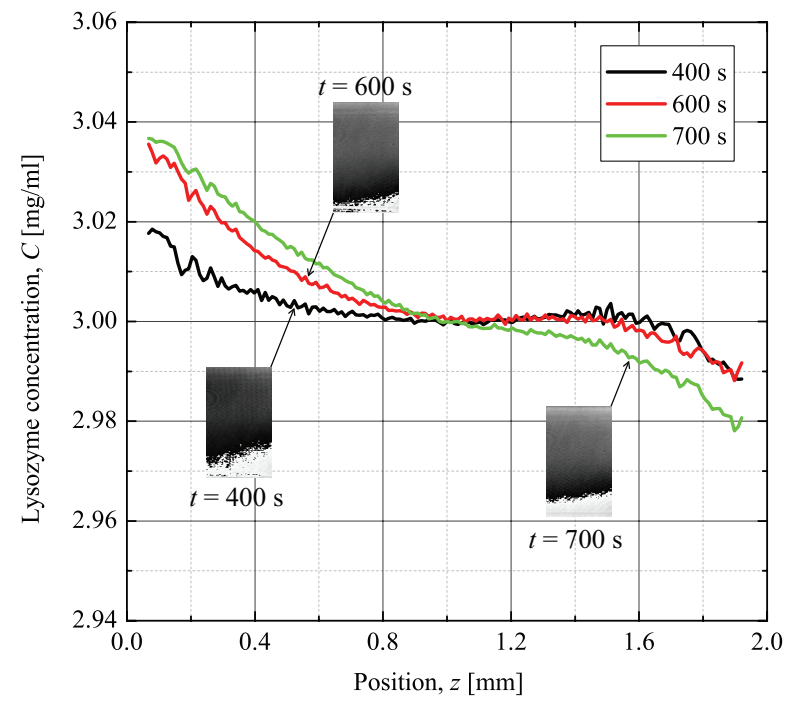

FIG. 18. Concentration profiles for the thermodiffusion phase of a lysozymewater binary solution $(3 \mathrm{mg} / \mathrm{ml})$. The temperature difference is set to $\Delta T$ $=5^{\circ} \mathrm{C}$ between the liquid/copper boundaries separated by $h=2 \mathrm{~mm}$. The concentration difference $\Delta C$ is too small to obtain a reliable measurement of the Soret coefficient. Nevertheless, the thermophobic nature $\left(S_{T}>0\right)$ of the lysozyme molecules is evident from this experimental result.

$\left(C_{m}=3 \mathrm{mg} / \mathrm{ml}\right)$ and the phase-shifted data corresponding to each profile. The distance between the walls was set to $h=2$ $\mathrm{mm}$, the temperature difference to $\Delta T=5^{\circ} \mathrm{C}$, and the mean temperature to $T_{m}=25^{\circ} \mathrm{C}$. The beam deflection problem is not clearly visible with this temperature difference, but still, the data near the heated copper/liquid boundaries could not be retrieved. Furthermore, the concentration difference $\Delta C$ between the upper and lower boundaries is too small to obtain a reliable measurement of the Soret coefficient. The temporal fluctuation of $\Delta C$ with only one fringe in the phase-shifted data is too large, so the linear concentration profile is not reliable and therefore this result is not included in Fig. 18. Therefore, the Soret coefficient is nearly zero at $C_{m}=3 \mathrm{mg} / \mathrm{ml}$ and $T_{m}=25^{\circ} \mathrm{C}$. We can also observe that the lysozyme molecules $(14.3 \mathrm{kDa})$ are thermophobic $\left(S_{T}>0\right)$ in this dilute aqueous solution because they migrate from the upper hot region to the lower cold region. This result is in accordance with those reported by Piazza et al. ${ }^{15}$ where the Soret coefficient is nearly zero, but positive, for a dilute lysozyme solution $\left(C_{m}=10 \mathrm{mg} / \mathrm{ml}\right)$ at $T=25^{\circ} \mathrm{C}$ and $\mathrm{pH}=7.1$ in the presence of $100 \mathrm{mM} \mathrm{NaCl}$ (which is used to screen the electrostatic effects at lower ionic strengths).

\section{CONCLUSIONS}

In this study, we have proposed a novel experimental technique to visualize thermodiffusion and measure the Soret coefficient in transparent liquid binary solutions. The measurement apparatus consists of two orthogonally aligned phase-shifting interferometers using a single rotating polarizer that is synchronized with a CCD camera. The phaseshifted data is processed in real-time by using a temporal phase-shifting algorithm. A Soret cell $(\mathrm{W} \times \mathrm{L} \times \mathrm{H})=(10$ 
$\times 20 \times 1-2) \mathrm{mm}^{3}$ was built with lateral transparent walls made of quartz glass and upper and lower boundaries made of copper. The transparent walls permit the measurement of diffusion from two orthogonal views, while the metallic walls allow a rapid temperature control at the copper/liquid boundaries. The temperature control is conducted with Peltier modules regulated with a PID system. Thermodiffusion experiments were conducted with the height of the cell set to $1.5 \mathrm{~mm}$ and $2 \mathrm{~mm}$. This is much less than the height of the cell used by previous research groups that use interferometric methods. ${ }^{20,34} \mathrm{~A}$ shorter cell height has three main advantages:

(1) Reducing the volume sample (directly proportional to the height). This is useful when measuring the thermophysical properties of expensive proteins, such as aprotinin.

(2) Shortening the measurement time (directly proportional to the second power of the height). This is necessary to measure thermodiffusion of biomolecules that have the risk of conformational changes. A shorter measurement time is also desirable to procure a more time-efficient measurement suitable for industrial applications.

(3) Increasing the hydrodynamic stability of the system (directly proportional to the third power of the height). This is particularly important when measuring negative Soret coefficient, which are known to cause Soret driven instabilities. ${ }^{47}$

The main disadvantage of having a shorter cell height is the increase of the error due to the beam deflection problem. A careful alignment of the cell with the test beam of the interferometer can reduce this problem.

The measurement technique was first validated by measuring the Soret coefficient of two well-known liquid pairs: ethanol-water (ethanol 39.12 wt.\%) and isobutylbenzenedodecane (50.0 wt.\%), the latter being one of the binary mixtures in the Fontainebleau benchmark. ${ }^{12}$ Preliminary experiments were conducted to measure the contrast factor of each liquid pair. These contrast factors serve as a link between the unwrapped phase-shifted data and the concentration, i.e., the measurement technique is independent of reference contrast factors found in the literature. The measured Soret coefficients, isothermal diffusion coefficients, and thermodiffusion coefficients for the two benchmark mixtures are in good agreement with the literature. Moreover, the measurement time of the Soret coefficient for the liquid pairs was less than four hours. This is about $1 / 3$ of the measurement time reported in recent studies that use similar interferometric techniques. ${ }^{19}$ The temporal phase-shifting technique allows us to determine the phase map (phase-shifted data) in quasi-real time. This image processing technique is a versatile method that can be used as an alternative method to the FFT method previously reported. ${ }^{19}$ However, the optical setup that we propose, which comprises a rotating polarizer, is more complex than the conventional Mach-Zehnder interferometer used for FFT methods. This is mainly due to the rigorous alignment of the linearly polarized beam in the polarizing Mach-Zehnder interferometer and the synchronization of the rotation polarizer with the CCD camera.
The measurement technique was then applied to two diluted protein aqueous solutions at $3 \mathrm{mg} / \mathrm{ml}$ : aprotinin-water and lysozyme-water. It was found that the aprotinin molecules have a thermophilic behavior $\left(S_{T}<0\right)$ in its highly diluted form, whereas lysozyme molecules have a thermophobic behavior $\left(S_{T}>0\right)$ at the same concentration level. It was also shown that thermodiffusion for the two diluted protein aqueous solutions is weaker than for the benchmark mixtures, i.e., few fringes were detected in the phase-shifted data. In order to bring the concentration field to a value that could be measured by using our optical apparatus, a higher temperature difference was applied to the aprotinin-water solution. However, it was shown that the increment of the temperature difference also worsens the beam deflection problem, making unfeasible the measurement of Soret coefficient values only from the thermodiffusion phase. Then, the isothermal diffusion phase was used to estimate the concentration difference in the steady state of the thermodiffusion phase and, thus, a reliable Soret coefficient for the aprotinin molecules was determined in the aqueous solution $(3 \mathrm{mg} / \mathrm{ml})$. In contrast, the concentration difference for the lysozyme-water binary solution $(3 \mathrm{mg} / \mathrm{ml})$ was too small to measure the Soret coefficient of the lysozyme component, i.e., the Soret coefficient was almost zero. This result is in accordance with that reported by Piazza et al. ${ }^{15}$ where the Soret coefficient of lysozyme in a dilute solution $(10 \mathrm{mg} / \mathrm{ml})$ at $T=25^{\circ} \mathrm{C}$ and $\mathrm{pH}=7.1$ was found to be almost zero. In spite of the weak thermodiffusion effect, our results show that the thermophobic or thermophilic behavior of the proteins molecules could be determined by using phase-shifting interferometry.

The proposed optical technique, called orthogonal phaseshifting interferometry, was used to visualize for the first time thermodiffusion of protein molecules. This optical method can be used as a tool to deepen our understanding of thermodiffusion in biological systems, and specifically of thermophoresis of protein and DNA molecules.

\section{ACKNOWLEDGMENTS}

This research was funded by the Grant-in-Aid for Young Scientists (A) (Grant No. 21686020) of The Japanese Ministry of Education, Culture, Sports, Science and Technology (MEXT). We would like to extend our utmost gratitude to the Tohoku University Global COE program for the financial support, as well as to the administration of École Centrale de Lyon and Tohoku University for making possible the collaboration on the joint double-degree Ph.D. program.

\section{APPENDIX: PHASE-SHIFTING EQUATION}

In this study, we have chosen a three-bucket, or threestep, temporal phase-shifting technique ${ }^{50}$ to obtain highresolution phase-shifted data in the isothermal diffusion and thermodiffusion experiments. A rotating polarizer with transmission angle $\theta$ was placed before two CCD cameras (for $L 1$ and $L 2$ ) in the polarizing Mach-Zehnder interferometer described in the text. In this appendix, we will derive the phase-shifting equation for the interferometer that uses the 
beam $L 1$, in which the linear polarization of the test beam and reference beam is 0 and $\pi / 2$, respectively, as shown in Fig. 4.

The amplitude $A(y, z)$ of the light $L 1$ passing through a rotating polarizer with a transmission angle $\theta$ is

$$
A(y, z)=A_{r} \cos (\omega t-\theta)+A_{o} \cos (\omega t+\theta+\varphi),
$$

where $\varphi, \omega, A_{r}$, and $A_{o}$ are the phase shift, the angular frequency of the circularly polarized light, the amplitudes of the reference beam, and the amplitude of the test (or object) beam, respectively. The intensity $I(y, z)$ of light acquired by each CCD camera is then equal to the time-averaged absolute value of the amplitude described in Eq. (A1). Therefore, the intensity for a period $P$ is

$$
I(y, z)=\frac{1}{P} \int_{0}^{P}|A(\theta, \varphi)|^{2} d t,
$$

where $P=2 \pi / \omega$. After expanding arithmetically and simplifying Eq. (A2), $I(y, z)$ becomes

$$
I(y, z)=\frac{1}{2}\left(A_{r}^{2}+A_{o}^{2}\right)+A_{r} A_{o} \cos (\varphi+2 \theta) .
$$

Therefore, the spatial intensity $I(y, z)$ depends on both the transmission angle $\theta$ and the phase shift $\varphi$; the former is controlled by a stepper motor and the latter is the physical quantity to be measured. Creath ${ }^{50}$ has described the threebucket phase-shifting with a constant $\Delta \theta$ value, in particular for $\Delta \theta=\pi / 2$ and $2 \pi / 3$. We consider that $\Delta \theta=2 \pi / 3$ is the best choice in order to simplify the synchronization procedure of the rotating polarizer and the CCD cameras. The phase-shifting interferograms $I_{1}(y, z), I_{2}(y, z), I_{3}(y, z)$ that are acquired at $\theta=0,2 \pi / 3,4 \pi / 3$, respectively, become

$$
\begin{aligned}
& I_{1}(y, z)=\frac{1}{2}\left(A_{r}^{2}+A_{o}^{2}\right)+A_{r} A_{o} \cos (\varphi), \\
& I_{2}(y, z)=\frac{1}{2}\left(A_{r}^{2}+A_{o}^{2}\right)+A_{r} A_{o} \cos \left(\varphi+\frac{4 \pi}{3}\right), \\
& I_{3}(y, z)=\frac{1}{2}\left(A_{r}^{2}+A_{o}^{2}\right)+A_{r} A_{o} \cos \left(\varphi+\frac{8 \pi}{3}\right) .
\end{aligned}
$$

Here, we assume that the phase shift $\varphi$ is constant for the three consecutive intensities $I_{1}, I_{2}$, and $I_{3}$, i.e., the technique is only applicable for a steady state phenomenon or for phenomena where $\varphi$ does not change significantly between $I_{l}$, $I_{2}$, and $I_{3}$. The amplitudes $A_{r}$ and $A_{o}$ in Eq. (A5) can be cancelled by using basic algebraic operations. The phase-shifted data (phase map) is then

$$
\varphi(y, z)=\arctan \left(\sqrt{3} \frac{I_{2}(y, z)-I_{3}(y, z)}{I_{2}(y, z)-2 I_{1}(y, z)+I_{3}(y, z)}\right) .
$$

This phase-shifting equation is also valid for the interferometer that uses L2. Equation (A5) was used in this study to determine the concentration profiles within a diffusion or Soret cell. These concentration profiles are then used to determine the Soret coefficients in organic binary solutions. Because of the nature of the arctangent calculation in Eq. (A5), the phase calculation is done modulo $\pi$ (between $-\pi / 2$ and $+\pi / 2$ ). The phase shift can then be subsequently adjusted by examining the values of $\sin \varphi$ and $\cos \varphi$.
${ }^{1}$ C. Ludwig, Sitzungsber. Akad. Wiss. Wien, Math.-Naturwiss. Kl., Abt. 2A 20, 539 (1856).

${ }^{2}$ C. Soret, Arch. Sci. Phys. Nat. 2, 48 (1879).

${ }^{3}$ T. Graham, Philos. Trans. R. Soc. London 140, 1 (1850).

${ }^{4}$ T. Graham, Philos. Trans. R. Soc. London 151, 183 (1861).

${ }^{5}$ A. Fick, Ann. Phys. (Berlin) 170, 59 (1855).

${ }^{6}$ F. Montel, J. Bickert, A. Lagisquet, and G. Galliéro, J. Pet.. Sci. Eng. 58, 391 (2007).

${ }^{7}$ R. Piazza and A. Parola, J. Phys.: Condens. Matter 20, 153102 (2008).

${ }^{8}$ D. Braun and A. Libchaber, Phys. Rev. Lett. 89, 188103 (2002).

${ }^{9}$ C. J. Wienken, P. Baaske, U. Rothbauer, D. Braun, and S. Duhr, Nat. Commun. 1, 100 (2010).

${ }^{10}$ M. Jerabek-Willemsen, C. J. Wienken, D. Braun, P. Baaske, and S. Duhr, Assay Drug Dev. Technol. 9, 342 (2011).

${ }^{11}$ J. K. Platten, J. Appl. Mech. 73, 5 (2006).

${ }^{12}$ J. K. Platten, M. M. Bou-Ali, P. Costesèque, J. F. Dutrieux, W. Köhler, C. Leppla, S. Wiegand, and G. Wittko, Philos. Mag. 83, 1965 (2003).

${ }^{13}$ A. Königer, B. Meier, and W. Köhler, Philos. Mag. 89, 907 (2009).

${ }^{14}$ S. Iacopini and R. Piazza, Europhys. Lett. 63, 247 (2003).

${ }^{15}$ R. Piazza, S. Iacopini, and B. Triulzi, Phys. Chem. Chem. Phys. 6, 1616 (2004).

${ }^{16}$ A. Königer, H. Wunderlich, and W. Köhler, J. Chem. Phys. 132, 174506 (2010).

${ }^{17}$ P. Bordat, D. Reith, and F. Müller-Plathe, J. Chem. Phys. 115, 8978 (2001).

${ }^{18}$ P. Kolodner, H. Williams, and C. Moe, J. Chem. Phys. 88, 6512 (1988).

${ }^{19}$ A. Mialdun and V. Shevtsova, J. Chem. Phys. 134, 044524 (2011).

${ }^{20}$ J. Colombani, H. Dez, J. Bert, and J. Dupuy-Philon, Phys. Rev. E 58, 3202 (1998).

${ }^{21}$ J. K. Platten, J. F. Dutrieux, and G. Chavepeyer, Thermal Nonequilibrium Phenomena in Liquid Mixtures (Springer-Verlag, 2002), pp. 313-333.

${ }^{22}$ M. M. Bou-Ali, O. Ecenarro, J. Madariaga, C. Santamaria, and J. Valencia, J. Phys.: Condens. Matter 10, 3321 (1998).

${ }^{23}$ J. F. Dutrieux, J. K. Platten, G. Chavepeyer, and M. M. Bou-Ali, J. Phys. Chem. B 106, 6104 (2002).

${ }^{24}$ E. Cussler, Diffusion: Mass Transfer in Fluid Systems, 3rd ed. (Cambridge University Press, 2009).

${ }^{25}$ A. Mialdun, V. Yasnou, V. Shevtsova, A. Königer, W. Köhler, D. Alonso de Mezquia, and M. M. Bou-Ali, J. Chem. Phys. 136, 244512 (2012).

${ }^{26}$ V. V. Sechenyh, J. C. Legros, and V. Shevtsova, J. Chem. Thermodyn. 43, 1700 (2011).

${ }^{27}$ W. Köhler, J. Chem. Phys. 98, 660 (1993).

${ }^{28} \mathrm{~S}$. Wiegand, Thermal Nonequilibrium Phenomena in Liquid Mixtures (Springer, 2002), pp. 189-210.

${ }^{29}$ H. Cabrera, E. Sira, K. Rahn, and M. García-Sucre, Appl. Phys. Lett. 94, 051103 (2009).

${ }^{30}$ H. Cabrera, L. Martí-López, E. Sira, K. Rahn, and M. García-Sucre, J. Chem. Phys. 131, 031106 (2009).

${ }^{31}$ B.-J. de Gans, R. Kita, B. Müller, and S. Wiegand, J. Chem. Phys. 118, 8073 (2003)

${ }^{32}$ S. Wiegand, H. Ning, and H. Kriegs, J. Phys. Chem. B 111, 14169 (2007).

${ }^{33}$ Y. Kishikawa, S. Wiegand, and R. Kita, Biomacromolecules 11, 740 (2010).

${ }^{34}$ A. Mialdun and V. Shevtsova, Int. J. Heat Mass Transfer 51, 3164 (2008).

${ }^{35}$ A. Mialdun and V. Shevtsova, C. R. Mec. 339, 362 (2011).

${ }^{36}$ M. Takeda, H. Ina, and S. Kobayashi, J. Opt. Soc. Am. 72, 156 (1982).

${ }^{37}$ V. Shevtsova, Adv. Space Res. 46, 672 (2010).

${ }^{38}$ V. Shevtsova, T. Lyubimova, Z. Saghir, D. Melnikov, Y. Gaponenko, V. Sechenyh, J. C. Legros, and A. Mialdun, J. Phys.: Conf. Ser. 327, 012031 (2011).

${ }^{39}$ Z. Guo, S. Maruyama, and A. Komiya, J. Phys. D 32, 995 (1999).

${ }^{40}$ S. Maruyama, T. Shibata, and K. Tsukamoto, Exp. Therm. Fluid Sci. 19, 34 (1999).

${ }^{41}$ A. Komiya and S. Maruyama, Exp. Therm. Fluid Sci. 30, 535 (2006).

${ }^{42}$ J. F. Torres, A. Komiya, E. Shoji, J. Okajima, and S. Maruyama, Opt. Lasers Eng. 50, 1287 (2012).

${ }^{43}$ J. F. Torres, A. Komiya, J. Okajima, and S. Maruyama, Defect. Diffus. Forum 326-328, 452 (2012)

${ }^{44}$ A. Komiya, J. F. Torres, and S. Maruyama, Defect. Diffus. Forum 297-301, 624 (2010).

${ }^{45}$ D. Hurle and E. Jakeman, J. Fluid Mech. 47, 667 (1971).

${ }^{46} \mathrm{G}$. Zimmermann and U. Müller, Int. J. Heat Mass Transfer 35, 2245 (1992).

${ }^{47}$ D. Melnikov, A. Mialdun, and V. Shevtsova, J. Non-Equil. Thermody. 32, 259 (2007). 
${ }^{48}$ H. Takeda, S. Maruyama, J. Okajima, S. Aiba, and A. Komiya, Cryobiology 59, 275 (2009).

${ }^{49}$ M. Hipp, J. Woisetschläger, P. Reiterer, and T. Neger, Measurement 36, 53 (2004).

${ }^{50}$ K. Creath, Progress in Optics (Elsevier, 1988), pp. 349-393.

${ }^{51}$ J. Colombani and J. Bert, J. Mol. Liq. 134, 8 (2007).
${ }^{52}$ Y. Duan and P. A. Kollman, Science 282, 740 (1998).

${ }^{53}$ D. Henry and H. Ben Hadid, Phys. Rev. E 76, 016314 (2007).

${ }^{54}$ R. J. Jiménez Riobóo, M. Philipp, M. A. Ramos, and J. K. Krüger, Eur. Phys. J. E 30, 19 (2009).

${ }^{55}$ M. Capobianchi, T. F. Irvine, Jr., N. K. Tutu, and G. Alanson Greene, Exp. Therm. Fluid Sci. 18, 33 (1998). 